TRANSACTIONS OF THE

AMERICAN MATHEMATICAL SOCIETY

Volume 251, July 1979

\title{
THE ANALYTIC CONTINUATION OF THE DISCRETE SERIES. II
}

BY

NOLAN R. WALLACH

\begin{abstract}
This is the second in a series of papers on the analytic continuation of the holomorphic discrete series. In this paper necessary and sufficient conditions for unitarizability are given in the case of line bundles. The foundations for the vector valued case are begun.
\end{abstract}

1. Introduction. In Wallach [8] it was shown that the representations of the holomorphic discrete series of a simply connected, connected, simple Lie group, $G$, "analytically continue" to give new unitary representations. In Wallach [8] the extent of this continuation was determined for the universal covering group of $S U(n, 1)$ and the holomorphic discrete series of $S U(n, 1)$ corresponding to homogeneous line bundles over the unit ball in $C^{n}$. In this paper we solve the same problem for arbitrary $G$ (as above) admitting holomorphic discrete series. We note that the sufficiency of the condition of Corollary 5.11 has been proved by Rossi and Vergue by very different methods (using techniques of [6]). They also conjectured the necessity.

This paper is quite algebraic in nature. It is more in the spirit of HarishChandra [1] than the first paper in this series. In this paper we complete the circle of ideas of Harish-Chandra [1] by giving a proof of Theorem 3 of [1] which is in the spirit of [1]. In [1] a proof is not given. It is deferred to [2] where the full theory of the holomorphic discrete series is used.

One of the main motivations for studying the analytic continuation of the holomorphic discrete is to prove vanishing theorems for $(0, p)$ cohomology of compact, locally symmetric, Kähler manifolds. (See Hotta-Wallach [4].) That is if a certain value of the continuing parameter does not correspond to a unitary representation then a $(0, p)$ Betti number is zero. Of course, one needs to study more general holomorphic discrete series than is studied in this paper. The general case will be studied in later papers in this series.

2. Some structural lemmas. Let $g$ be a simple Lie algebra over the reals $(\mathbf{R})$. Let $\mathfrak{g}=\mathfrak{f} \oplus \mathfrak{p}$ be a Cartan decomposition of $\mathfrak{g}$ (cf. Helgason [3] or Wallach [9]). We assume that $[\mathfrak{f}, \mathfrak{f}]=\mathfrak{f}_{1} \neq \mathfrak{f}$. If $\mathfrak{h}_{*} \subset \mathfrak{f}$ is a maximal abelian subalgebra

Received by the editors June 16, 1975.

AMS (MOS) subject classifications (1970). Primary 17B10, $20 \mathrm{G} 05$.

Key words and phrases. Representation, semisimple Lie algebra, semisimple Lie group, irreducibility, unitarizability, holomorphic discrete series, highest weight. 
of $\mathfrak{f}$ then $\mathfrak{h}_{*}$ is a Cartan subalgebra of $\mathfrak{g}$. Let $\mathfrak{g}_{c}, \mathfrak{f}_{c}, \mathfrak{p}_{c}, \mathfrak{h}$ denote the respective complexifications of $\mathfrak{g}, \mathfrak{f}, \mathfrak{p}$ and $\mathfrak{h}_{*}$. Let $H \in \mathfrak{h}$ be such that $\mathfrak{f}=\mathbf{R} i H \oplus \mathfrak{f}_{1}$. Then (as is well known, see Helgason [3]) we may assume that ad $\left.H\right|_{\mathfrak{p}_{c}}$ has two eigenvalues \pm 1 . Let $\Delta$ be the root system of $\left(\mathfrak{g}_{c}, \mathfrak{h}\right)$. Let $\Delta_{K}=\left\{\alpha \in \Delta \mid \mathfrak{g}_{\alpha}\right.$ $\left.\subset \mathfrak{f}_{c}\right\}\left(\mathfrak{g}_{\alpha}=\left\{X \in \mathfrak{g}_{c} \mid(h, X]=\alpha(h) X\right.\right.$ for $\left.\left.h \in \mathfrak{h}\right\}\right)$. Then $\Delta_{K}=\{\alpha \in \Delta \mid \alpha(H)$ $=0\}$. Let $\Delta_{P}=\left\{\alpha \in \Delta \mid \mathfrak{g}_{\alpha} \subset \mathfrak{p}_{c}\right\}$.

Let $H_{1}=H, H_{2}, \ldots, H_{l}$ be a basis of $\mathfrak{h}_{\mathbf{R}}=\{h \in \mathfrak{h} \mid \alpha(h) \in \mathbf{R}$ for all $\alpha \in \Delta$ \}. Order $\mathfrak{h}_{\mathbb{R}}^{*}$ lexicographically relative to this ordered basis. Let $\Delta^{+}$be the corresponding positive roots. Let $\Delta_{K}^{+}=\Delta^{+} \cap \Delta_{K}, \Delta_{P}^{+}=\Delta^{+} \cap \Delta_{P}$. Let $\gamma_{1}, \ldots, \gamma_{r}$ be constructed as in Harish-Chandra [2] (see also Helgason [3] or Wallach [9]). In particular $\gamma_{1}, \ldots, \gamma_{r}$ satisfy:

(i) $\gamma_{1}$ is the smallest element of $\Delta_{P}^{+}$and $\gamma_{1}<\cdots<\gamma_{r}$ and $\gamma_{i} \pm \gamma_{j} \in \Delta$ for all $1<i<j \leqslant r$.

(ii) $r$ is the split rank of $g$.

(iii) Let $B$ be the Killing form of $g_{c}$.

If $\lambda \in \mathfrak{h}^{*}$ let $H_{\lambda} \in \mathfrak{h}$ be defined by $B\left(H_{\lambda}, h\right)=\lambda(h)$ for $h \in \mathfrak{h}$. Let $\mathfrak{h}^{-}=$ $\sum_{i=1}^{r} C H \gamma_{i}, \mathfrak{h}^{+}=\left\{h \in \mathfrak{h} \mid \gamma_{i}(h)=0, i=1, \ldots, r\right\}$. If $\alpha \in \Delta_{K}^{+}$then $\left.\alpha\right|_{\mathfrak{h}^{-}}$is in one of the following possible forms:

(a) $\left.\alpha\right|_{\mathfrak{h}^{-}}=-\frac{1}{2} \gamma_{j}$ for some $1 \leqslant j \leqslant r$,

(b) $\left.\alpha\right|_{\mathfrak{h}^{-}}=\frac{1}{2}\left(\gamma_{i}-\gamma_{j}\right)$ for some $1 \leqslant j<i \leqslant r$,

(c) $\left.\alpha\right|_{\mathfrak{h}^{-}}=0$.

(iv) If $\alpha \in \Delta_{P}^{+}$then $\left.\alpha\right|_{\mathfrak{h}^{-}}$is in one of the following possible forms:

(a) $\left.\alpha\right|_{\mathfrak{h}^{-}}=\frac{1}{2} \gamma_{i}$ for some $1 \leqslant i \leqslant r$,

(b) $\left.\alpha\right|_{\mathfrak{h}^{-}}=\frac{1}{2}\left(\gamma_{i}+\gamma_{j}\right)$ for some $1 \leqslant i \leqslant j \leqslant r$.

(v) If $\Delta_{0}=\left\{\alpha \in \Delta|\alpha|_{\mathfrak{h}^{-}}=0\right\}$ then $\Delta_{0} \subset \Delta_{K}$ (see (iii)). If $\alpha \in \Delta_{0}$ then $\alpha \pm \gamma_{i} \notin \Delta$ for any $i=1, \ldots, r$. If $\alpha \in \Delta$ then at most one of $\alpha \pm \gamma_{i}$ is in $\Delta$ for each $i$. In particular the $\gamma_{i}$ are all extreme for the adjoint representation of $\mathfrak{g}_{c}$ on $\mathfrak{g}_{c}$ hence $B\left(H \gamma_{i}, H \gamma_{i}\right)=B\left(H \gamma_{j}, H \gamma_{j}\right)$ for all $i, j$ (cf. Moore [5]).

For proofs of these results see Harish-Chandra [2].

Let $\mathfrak{p}^{+}=\Sigma_{\alpha \in \Delta_{p}^{+}} \mathfrak{g}_{\alpha}, \mathfrak{p}^{-}=\Sigma_{\alpha \in \Delta_{p}^{+}} \mathfrak{g}_{-\alpha}$. Then $\left[\mathfrak{p}^{+}, \mathfrak{p}^{+}\right]=\left[\mathfrak{p}^{-}, \mathfrak{p}^{-}\right]=0$ and $\operatorname{ad}\left(k_{c}\right) \mathfrak{p}^{-} \subset \mathfrak{p}^{-}$.

LeMma 2.1. Let $\Delta^{0}=\left\{\alpha \in \Delta|\alpha|_{\mathfrak{p}^{-}} \neq \pm \frac{1}{2} \gamma_{i}\right.$ for any $\left.i\right\}$.

(i) $\Delta^{0}$ is a subsystem of $\Delta$ (that is if $\alpha, \beta \in \Delta^{0}, \alpha+\beta \in \Delta$ then $\alpha+\beta \in \Delta^{0}$, if $\alpha \in \Delta^{0}, \alpha \in \Delta$ ).

(ii) If $\Phi \subset \Delta$ is a subsystem so that $\Delta^{0} \subset \Phi$ then $\Phi=\Delta^{0}$ or $\Phi=\Delta$.

Proof. Let $h_{i}=H_{\gamma_{i}} /\left\langle\gamma_{i}, \gamma_{i}\right\rangle$ (here $\langle\lambda, \mu\rangle=B\left(H_{\lambda}, H_{\mu}\right)$ for $\left.\lambda, \mu \in \mathfrak{h}^{*}\right)$. Let $H_{0}=\sum_{i=1}^{r} h_{i}, \alpha \in \Delta^{0}$ if and only if $\alpha\left(H_{0}\right)= \pm 1$ or $0 . \alpha \in \Delta-\Delta^{0}$ if and only if $\alpha\left(H_{0}\right)= \pm \frac{1}{2}$. This clearly implies (i).

Let $\eta=\exp \left(2 \pi i\right.$ ad $\left.H_{0}\right)$. Then $\eta$ is an involutive automorphism of $g_{c}$. If 
$\mathrm{g}_{c}^{+}=\left\{x \in \mathfrak{g}_{c} \mid \eta(x)=x\right\}$ then $\mathfrak{g}_{c}^{+}=\mathfrak{h}+\sum_{\alpha \in \Delta^{\circ}} \mathfrak{g}_{\alpha}$. (ii) now follows from Wallach [11]. Q.E.D.

Let $\left.\theta\right|_{\mathfrak{t}}=I,\left.\theta\right|_{\mathfrak{p}}=-I$. Let $\tilde{g}_{c}^{0}=\mathfrak{h}+\Sigma_{\alpha \in \Delta^{0}} \mathfrak{g}_{\alpha}$. Then $\theta \tilde{g}_{c}^{0} \subset \tilde{g}_{c}^{0}$. Let $\tilde{g}^{0}=$ $\mathfrak{g} \cap \tilde{\mathfrak{g}}_{c}^{0}$. Then $\tilde{\mathfrak{g}}^{0}$ is a real form of $\tilde{\mathfrak{g}}_{c}^{0}$. Let $\mathfrak{f}^{0}=\mathfrak{i} \cap \tilde{\mathfrak{g}}^{0}, \mathfrak{p}^{0}=\mathfrak{p} \cap \tilde{\mathfrak{g}}^{0}$.

LEMMA 2.2. Let $\mathrm{g}^{0}=\left[\mathfrak{p}^{0}, \mathfrak{p}^{0}\right] \oplus \mathfrak{p}^{0}$. Then $\mathrm{g}^{0}$ is a simple subalgebra of $\mathfrak{g}$. If $\mathfrak{f}^{0}=\left[\mathfrak{p}^{0}, \mathfrak{p}^{0}\right]$ then $\mathfrak{f}^{0}=\mathrm{g}^{0} \cap \mathfrak{f}^{0}$ and $\left[\mathfrak{f}^{0}, \mathfrak{l}^{0}\right] \neq \mathfrak{t}^{0}$. If $\mathrm{g}_{c}^{0}$ is the complexification of $\mathrm{g}^{0}$ then

(i) $\mathfrak{h}^{-} \subset \mathfrak{g}_{c}^{0}$.

(ii) $\mathfrak{p}_{c}^{0}=\mathfrak{p}_{0}^{+} \oplus \mathfrak{p}_{0}^{-}$with $\mathfrak{p}_{0}^{+}=\mathfrak{p}_{c}^{0} \cap \mathfrak{p}^{+}$and $\mathfrak{p}_{0}^{-}=\mathfrak{p}_{c}^{0} \cap \mathfrak{p}^{-}$.

(iii) If $\mathfrak{h}_{0}^{+}=\mathfrak{h}^{+} \cap \mathfrak{g}_{c}^{0}$ then $\mathfrak{h}_{0}^{+} \oplus \mathfrak{h}^{-}$is a Cartan subalgebra of $\mathfrak{g}_{c}^{0}$.

Proof. $\left[\tilde{\mathfrak{g}}_{c}^{0}, \tilde{\mathfrak{g}}_{c}^{0}\right]$ is semisimple (see the proof of Lemma 2.1). Since $\mathfrak{g}_{c}^{0}$ is an ideal of $\left[\tilde{\mathrm{g}}_{c}^{0}, \tilde{\mathrm{g}}_{c}^{0}\right], \mathfrak{g}_{c}^{0}$ is semisimple. Using the results of Moore [5] it is clear that the restricted root system of $g^{0}$ relative to a maximal abelian subalgebra of $\mathrm{g}^{0}$ is connected. Hence $\mathrm{g}^{0}$ is simple. The rest of the proof of this lemma is quite easy and left to the reader. Q.E.D.

LEMMA 2.3. If $\beta_{1}, \ldots, \beta_{j} \in \Delta_{P}^{+}$and if $\beta_{1}+\cdots+\beta_{j}=\gamma_{1}+\cdots+\gamma_{j}$ then $\beta_{i} \in \Delta_{p}^{+} \cap \Delta^{0}=\Delta_{p}^{0,+}$.

Proof. Suppose that the lemma is false. Then $\beta_{1}, \ldots, \beta_{s} \notin \Delta_{p}^{0,+}$, $\beta_{s+1}, \ldots, \beta_{j} \in \Delta_{p}^{0,+}$, and $s \geqslant 1$. Let $H_{0}$ be as in the proof of Lemma 2.1. Then $\left(\gamma_{1}+\cdots+\gamma_{j}\right)\left(H_{0}\right)=j$. But $\beta_{i}\left(H_{0}\right)=\frac{1}{2}$ for $j<s$. $\beta_{s+t}\left(H_{0}\right)=1$ for $t=1, \ldots, j-s$. Hence $\left(\beta_{1}+\cdots+\beta_{j}\right)\left(H_{0}\right)=\frac{1}{2} s+j-s$. This is impossible.

Let $\Delta_{j}=\left\{\alpha \in \Delta^{0}|\alpha|_{\mathfrak{h}-} \in \sum_{i=1}^{j} \mathbf{R} \gamma_{i}\right\}$. Let $\Delta_{j, p}=\Delta_{j} \cap \Delta_{p}$. Let $\sum_{\alpha \in \Delta_{j, p}} \mathfrak{g}_{\alpha}=$ $\mathfrak{p}_{c}^{j}$. Let $\mathfrak{g}_{j, c}=\left[\mathfrak{p}_{c}^{j}, \mathfrak{p}_{c}^{j}\right]+\mathfrak{p}_{c}^{j}$. Let $\mathfrak{g}_{j}=\mathfrak{g} \cap \mathfrak{g}_{j, c} . \mathfrak{f}_{j}=\mathfrak{l} \cap \mathfrak{g}_{j}, \mathfrak{p}_{j}=\mathfrak{p}_{c}^{j}$. $\mathfrak{f}_{j}$.

LEMMA 2.4. $\mathfrak{g}_{j}$ is simple. $\mathfrak{g}_{j}=\mathfrak{f}_{j} \oplus \mathfrak{p}_{j}$ is a Cartan decomposition and $\left[\mathfrak{f}_{j}, \mathfrak{f}_{j}\right] \neq$

Proof. This lemma is a simple consequence of results of Moore [5].

LEMMA 2.5. If $\beta_{1}, \ldots, \beta_{j} \in \Delta_{p}^{+}$and $\sum \beta_{i}=\sum_{i=1}^{j} \gamma_{i}$ then $\beta_{i} \in \Delta_{j p}^{+}=\Delta_{p}^{+} \cap$ $\Delta_{j, p}, i=1, \ldots, j$.

Proof. $\beta_{i} \in \Delta_{p}^{0,+}$ by Lemma 2.3. But then $\left.\beta_{i}\right|_{\mathfrak{h}^{-}}=\frac{1}{2}\left(\gamma_{t_{i}}+\gamma_{s_{i}}\right), \quad 1<t_{i}$, $s_{i}<r . \sum \beta_{i}=\sum_{i=1}^{j} \gamma_{i}$. Hence $t_{i}, s_{i}<j$. Q.E.D.

Let $\mathfrak{p}_{j}^{+}$and $\mathfrak{p}_{j}^{-}$denote $\mathfrak{p}_{j, c} \cap \mathfrak{p}^{+}$and $\mathfrak{p}_{j, c} \cap \mathfrak{p}^{-}$respectively.

3. A theorem of Schmid. We retain the notation of $\S 2$. Since $\left[\mathfrak{p}^{-}, \mathfrak{p}^{-}\right]=0$ we see that $U\left(\mathfrak{p}^{-}\right)$the universal enveloping algebra of $\mathfrak{p}^{-}$is isomorphic with the symmetric algebra on $\mathfrak{p}^{-}$. Hence $U\left(\mathfrak{p}^{-}\right)$is graded by degree. Let $U^{j}\left(\mathfrak{p}^{-}\right)$ be the homogeneous elements of degree $j . \mathfrak{f}_{c}$ acts on $\mathfrak{p}^{-}$by the adjoint representation, hence on $U\left(\mathfrak{p}^{-}\right)$by derivations still denoted ad. 
THEOREM 3.1 (W. SCHMID [7]). As a representation of $\mathfrak{f}_{c}, U^{j}\left(\mathfrak{p}^{-}\right)$splits into a direct sum $\sum m_{\lambda, j} \tau_{\lambda}\left(\tau_{\lambda}\right.$ the irreducible finite dimensional representation of $\mathfrak{f}_{c}$ with highest weight, $\lambda$, relative to $\Delta_{K}^{+}$) with $m_{\lambda_{j}}=0$ or 1 depending on whether or not $\lambda=-\sum_{k=1}^{r} m_{k} \gamma_{k}, m_{1}>m_{2}>\cdots>m_{r}>0, m_{i} \in \mathbf{Z}$ and $\sum m_{i}=j$.

Setting $\lambda_{j}=-\left(\gamma_{1}+\cdots+\gamma_{j}\right)$ then every $\mathfrak{f}_{c}$ highest weight in $U^{j}\left(\mathfrak{p}^{-}\right)$is of the form $\sum_{i=1}^{r} n_{i} \lambda_{i}$ with $n_{i}>0, n_{i} \in \mathbf{Z}$ and $\sum i n_{i}=j$.

Set $\mathrm{n}_{K}^{+}=\Sigma_{\alpha \in \Delta_{k}^{+}} \mathfrak{g}_{\alpha}$. Set $U\left(\mathfrak{p}^{-}\right)^{\mathfrak{n}_{K}^{+}}=\left\{u \in U\left(\mathfrak{p}^{-}\right) \mid \operatorname{ad}\left(\mathfrak{n}_{K}^{+}\right) \cdot u=0\right\}$. Set for $\lambda \in \mathfrak{h}^{*}, U\left(\mathfrak{p}^{-}\right)_{\lambda}=\left\{u \in U\left(\mathfrak{p}^{-}\right) \mid \operatorname{ad}(h) u=\lambda(h) u\right.$ for $\left.h \in \mathfrak{h}\right\}$. Then $U\left(\mathfrak{p}^{-}\right)^{\mathfrak{n}_{k}^{+}}=$ $\sum U\left(\mathfrak{p}^{-}\right)_{\lambda} \cap U\left(\mathfrak{p}^{-}\right)^{\mathfrak{n}_{k}^{+}}$and $\operatorname{dim} U\left(\mathfrak{p}^{-}\right)_{\lambda} \cap U\left(\mathfrak{p}^{-}\right)^{\mathfrak{n}_{\kappa}^{+}}=0$ or 1 . Let $u_{j}$ be a nonzero element of $U\left(\mathfrak{p}^{-}\right)^{\mathfrak{n}_{k}^{+}} \cap U\left(\mathfrak{p}^{-}\right)_{\lambda_{j}}$. Then the above theorem and observations imply:

COROLlaRY 3.2. $U\left(\mathfrak{p}^{-}\right)^{\mathrm{n}_{\kappa}^{+}}$is the subalgebra of $U\left(\mathfrak{p}^{-}\right)$generated by $u_{1}, \ldots, u_{r}$.

We also need

LEMMA 3.3. $u_{j} \in U\left(\mathfrak{p}_{0}^{-}\right)$(see Lemma 2.2 for notation).

Proof. $u_{j} \in U^{j}\left(\mathfrak{p}^{-}\right)^{\mathrm{n}_{k}^{+}} \cap U\left(\mathfrak{p}^{-}\right)_{\lambda_{j}}$. If $X_{\alpha} \in \mathrm{g}_{\alpha}-\{0\}$ has been chosen for each $\alpha \in \Delta$ then $U^{j}\left(\mathfrak{p}^{-}\right)_{\lambda}$ is spanned by the elements of the forms $X_{-\beta_{1}} X_{-\beta_{2}} \cdots X_{-\beta_{j}}, \sum_{i=1}^{j} \beta_{i}=\sum_{i=1}^{j} \gamma_{i}$. Lemma 2.3 now implies the result.

4. The representations $V^{\Lambda}$. We retain the notation of the previous sections. Let $\mathfrak{n}^{+}=\Sigma_{\alpha \in \Delta}+\mathfrak{g}_{\alpha}, \mathfrak{n}^{-}=\Sigma_{\alpha \in \Delta}+\mathfrak{g}_{-\alpha}$. Let $U=U\left(\mathfrak{g}_{c}\right)$ be the universal enveloping algebra of $g_{c}$. The Poincaré-Birkhoff-Witt theorem implies that $U=U(\mathfrak{h}) \oplus\left(\mathfrak{n}^{-} U+U \mathfrak{n}^{+}\right)$(here $U(\mathfrak{h})$ is the universal enveloping algebra of h). Let $P: U \rightarrow U(\mathfrak{h})$ be the corresponding projection. If $\Lambda \in \mathfrak{h}^{*}$ extend $\Lambda$ to a homomorphism of $U(\mathfrak{h})$ to $\mathbf{C}$ in the usual fashion. Let for $g \in U, \xi_{\Lambda}(g)=$ $\Lambda(P(g))$. If $f \in U^{*}$ and $x \in U$ define $(x \cdot f)(g)=f(g x)$. Then $U^{*}$ becomes a $U$-module. Let for $\Lambda \in \mathfrak{h}^{*}, V^{\Lambda}=U \cdot \xi_{\Lambda}$. Then $V^{\Lambda}$ is an irreducible $g_{c}$ module (see Wallach [10]). Let $\sigma$ be the conjugation of $g_{c}$ relative to $g$ (that is $\sigma(X+i Y)=X-i Y$ for $X, Y \in \mathfrak{g})$. Let $\eta: U \rightarrow U$ be defined by: $\eta(1)=1$, $\eta(X)=-\sigma(X), X \in g_{c}, \eta(x y)=\eta(y) \eta(x)$ for $x, y \in U$.

DEFINITION 4.1. A representation $(\pi, V)$ of $g_{c}$ is said to have highest weight $\Lambda$ if there is $v_{0} \in V, v_{0} \neq 0$ so that

(1) $\pi(h) v_{0}=\Lambda(h) v_{0}$ for $h \in \mathfrak{h}$,

(2) $\pi\left(\mathfrak{n}^{+}\right) v_{0}=0$,

(3) $\pi(U) v_{0}=V$.

LEMMA 4.2. Let $(\pi, V)$ be a representation of $g_{c}$ with highest weight $\Lambda$. If $\langle$, is a Hermitian form on $V$ so that

(1) $\left\langle v_{0}, v_{0}\right\rangle=1$ ( $v_{0}$ as in Definition 4.1).

(2) $\langle\pi(x) v, w\rangle=-\langle v, \pi(x) w\rangle, x \in \mathfrak{g}$. 
Then $\Lambda \in \mathfrak{h}_{\mathbf{R}}^{*}$ that is $\left.\Lambda\left(\mathfrak{h}_{\mathbf{R}}\right) \subset \mathbf{R}\right)$ and $\left\langle\pi(x) \cdot v_{0}, \pi(y) \cdot v_{0}\right\rangle=\xi_{\Lambda}(\eta(y) x)$, for $x, y \in U$. If $\Lambda \in \mathfrak{h}_{\mathbb{R}}^{*}$ then $\left\langle\pi(x) \cdot v_{0}, \pi(y) v_{0}\right\rangle=\xi_{\Lambda}(\eta(y) x)$ defines a form on $V^{\Lambda}$ satisfying (1), (2).

Proof. We note that $\eta\left(\mathfrak{n}^{+}\right)=\mathfrak{n}^{-}$and $\eta\left(\mathfrak{n}^{-}\right)=\mathfrak{n}^{+}$. Thus $P(\eta(u))=$ $\eta(P(u)), \quad u \in U$. If $h \in \mathfrak{h}_{\mathbf{R}}$ then $\eta(h)=\mathfrak{h} \quad\left(i \mathfrak{h}_{\mathbf{R}} \subset \mathfrak{g}\right)$. Hence $\Lambda(h)=\left\langle\pi(h) v_{0}, v_{0}\right\rangle=\left\langle v_{0}, \pi(h) v_{0}\right\rangle=\overline{\Lambda(h)}$ for $h \in \mathfrak{h}_{\mathbf{R}}$. Hence $\Lambda \in \mathfrak{h}_{\mathbf{R}}^{*}$. Now (2) implies $\left\langle\pi(x) v_{0}, \pi(y) v_{0}\right\rangle=\left\langle\pi(\eta(y) x) v_{0}, v_{0}\right\rangle$. But if $\alpha(z)=\left\langle\pi(z) v_{0}, v_{0}\right\rangle$ then $\alpha\left(z \mathfrak{n}^{+}\right)=\alpha\left(\mathfrak{n}^{-} z\right)=0$. Hence $\alpha(z)=\alpha(P(z))$. If $h \in U(\mathfrak{h})$ then $\pi(h) v_{0}$ $=\Lambda(h)$. Thus $\alpha(z)=\xi_{\Lambda}(z)$. But then $\left\langle\pi(x) v_{0}, \pi(y) v_{0}\right\rangle=\xi_{\Lambda}(\eta(y) x)$. The last statement follows from the fact that one can define a unique homomorphism of $g_{c}$ modules $V \rightarrow V^{\Lambda}$ by $x \cdot v_{0} \rightarrow x$ (cf. Wallach [9, Theorem 4.4.5]). Q.E.D.

In this paper we will study some very special $\Lambda$. In a later paper in this series we will study the most general $\Lambda$ for which the form defined in Lemma 4.1 can be positive definite.

Let $\Lambda_{1}$ be defined by

(a) $\Lambda_{1}\left(H_{\alpha}\right)=0, \alpha \in \Delta_{K}$,

(b) $2\left\langle\Lambda_{1}, \gamma_{1}\right\rangle /\left\langle\gamma_{1}, \gamma_{1}\right\rangle=1$.

We will be studying only $\Lambda$ of the form $z \Lambda_{1}$ with $z \in C$.

We note that $V^{\Lambda}=U \cdot \xi_{\Lambda}$. Since $\mathfrak{n}^{+} \xi=0$ and $U(\mathfrak{h}) \xi=C \xi$ we see that $V^{\Lambda}=U\left(\mathfrak{n}^{-}\right) \xi_{\Lambda^{\prime}}$. If $\Lambda=z \Lambda_{1}$ then since $\mathfrak{f}_{c}=\mathfrak{n}_{k}^{-} \oplus \mathfrak{h} \oplus \mathfrak{n}_{k}^{+}$we see that $\xi_{* z \Lambda_{1}} l_{U\left(t_{c}\right)}$ is the corresponding " $\xi_{\Lambda}$ " for $\mathfrak{f}_{c}$. Hence $U\left(\mathfrak{f}_{c}\right) \cdot \xi_{z \Lambda_{1}}$ is irreducible. But by definition of $\Lambda_{1}$ the irreducible representation of $\mathfrak{f}_{c}$ with highest weight $z \Lambda_{1}$ is one dimensional. Hence $V^{z \Lambda_{1}}=U\left(\mathfrak{p}^{-}\right) \xi_{z \Lambda_{1}}$.

Let $u_{1}, \ldots, u_{r}$ be as in $\$ 3$. Let $U^{k_{1}}, \ldots, k_{r}\left(\mathfrak{p}^{-}\right)=\operatorname{ad}\left(U\left(\mathfrak{f}_{c}\right)\right) u_{1}^{k_{1}} \cdots u_{r}^{k_{r}}$. Then $U\left(\mathfrak{p}^{-}\right)=\Sigma_{k_{1}>0, k_{i} \in \mathbf{Z}} U^{k_{1}, \ldots, k_{r}\left(\mathfrak{p}^{-}\right)}$and $U^{k_{1}, \ldots, k_{r}\left(\mathfrak{p}^{-}\right)}$is the irreducible representation of $\mathfrak{f}_{c}$ with highest weight $\sum k_{i} \lambda_{i}$. By the above $V^{z \Lambda_{1}}=$ $\sum U^{k_{1}, \ldots, k_{r}}\left(\mathfrak{p}^{-}\right) \xi_{z} \Lambda_{1}$ and $U^{k_{1}}, \ldots, k_{r}\left(\mathfrak{p}^{-}\right) \xi_{z \Lambda_{1}}$ is the irreducible $\mathfrak{f}_{c}$ representation

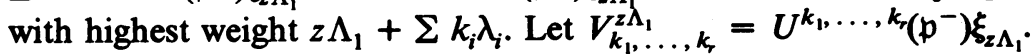

LEMMA 4.3. A necessary and sufficient condition for $V_{k_{1}, \ldots, k_{r}}^{z \Lambda_{1}} \neq 0$ is that $P_{k_{1}, \ldots, k_{r}}(z)=\xi_{z \Lambda_{1}}\left(\eta\left(u_{1}^{k_{1}} \cdots u_{r}^{k_{r}}\right) u_{1}^{k_{1}} \cdots u_{r}^{k_{r}}\right) \neq 0$.

Proof. Let $\mu$ be the anti-automorphism of $U$ defined by $\mu(x y)=$ $\mu(y) \mu(x), \mu(1)=1, \mu(x)=-x, x \in g_{c}$. Let $\{x, y\}=\xi_{z \Lambda_{1}}(\mu(y) x)$. Then $\{X u, v\}=-\{u, X v\}$ for $X \in g_{c}$. It is easily seen that the dual representation of $k_{c}$ on $U^{k_{1}}, \ldots, k_{r}\left(\mathfrak{p}^{-}\right)$is $\eta\left(U^{k_{1}}, \ldots, k_{r}\left(\mathfrak{p}^{-}\right)\right)$. Hence $\{$,$\} sets up a \mathfrak{f}_{c}$ invariant pairing of $\eta\left(U^{k_{1}, \ldots, k_{r}}\left(\mathfrak{p}^{-}\right)\right)$and $U^{k_{1}, \ldots, k_{r}}\left(\mathfrak{p}^{-}\right)$. Now the irreducibility implies that

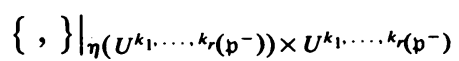


is either zero or a nonzero multiple of the canonical pairing. But then

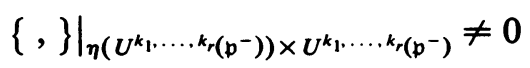

if and only if $P_{k_{1}, \ldots, k_{r}}(z) \neq 0$. In particular, we see that if $P_{k_{1}, \ldots, k_{r}}(z) \neq 0$ then $V_{k_{1}, \ldots, k_{r}}^{z \Lambda_{1}} \neq 0$ and if $P_{k_{1}, \ldots, k_{r}}(z)=0$ then $\left\{U\left(\mathfrak{p}^{+}\right), U^{k_{1}, \ldots, k_{r}}\left(\mathfrak{p}^{-}\right)\right\}=0$. But if $x, y \in U$ then $\left\{x \mathfrak{n}^{-}, y\right\}=0$. Arguing as above we find $\left\{x\left[\mathfrak{f}_{c}, \mathfrak{f}_{c}\right]\right.$, $y\}=0$. Thus if $\left\{U\left(\mathfrak{p}^{+}\right), y\right\}=0, y \cdot \xi_{z \Lambda_{1}}=0$. Hence if $P_{k_{1}, \ldots, k_{r}}(z)=0$ then $u_{1}^{k_{1}} \cdots u_{r}^{k_{r}} \cdot \xi_{z \Lambda_{1}}=0$. Q.E.D.

\section{Infinitesimally unitary modules.}

Definition 5.1. Let $(\pi, V)$ be a representation of $g_{c}$. Then $(\pi, V)$ is said to be $g$-infinitesimally unitary if there is a Hermitian positive definite inner product $\langle$,$\rangle on V$ so that $\langle\pi(x) v, w\rangle=-\langle v, \pi(x) w\rangle$ for $x \in g$.

LEMMA 5.2. There exists a representation of $\mathfrak{g}_{c}$ with highest weight $\Lambda$ which is $\mathrm{g}$-infinitesimally unitary if and only if $V^{\Lambda}$ is $\mathrm{g}$-infinitesimally unitary. $V^{z \Lambda_{1}}$ is $g$-infinitesimally unitary if and only if $z \in \mathbf{R}$ and $P_{k_{1}, \ldots, k_{r}}(z) \geqslant 0$ for all $k_{1}, \ldots, k_{r} \in \mathbf{Z}, k_{i} \geqslant 0$.

Proof. The first statement is an immediate consequence of Lemma 4.2. The second is a consequence of the proof of Lemma 4.3.

We now come to the critical observation.

LEMMA 5.3. $V^{z \Lambda_{1}}$ is g-infinitesimally unitary if and only if the irreducible representation of $\mathrm{g}^{0}$ with highest weight $z \lambda_{1}$ defined with $\mathrm{g}$ replaced by $\mathrm{g}^{0}$ is $\mathfrak{g}^{0}$-infinitesimally unitary.

Proof. $u_{j} \in U\left(\mathfrak{p}_{0}^{-}\right)$and $\mathfrak{g}_{c}^{0}=\left(\mathfrak{n}^{-} \cap \mathfrak{g}_{c}^{0}\right) \oplus\left(\mathfrak{h} \cap \mathfrak{g}_{c}^{0}\right) \oplus\left(\mathfrak{n}^{+} \cap \mathfrak{g}_{c}^{0}\right)$. Thus the functions $P_{k_{1}, \ldots, k_{r}}(z)$ are computed in $U\left(g_{c}^{0}\right)$. Q.E.D.

Let $\mathfrak{g}_{u}=\mathfrak{f}+i \mathfrak{p}$. Let $\tau$ be conjugation in $\mathfrak{g}_{c}$ relative to $g_{u}$. Let $\left\{E_{\alpha}\right\}$ be a Weyl basis of $\mathfrak{g}_{c}$ relative to $\left\{\mathfrak{g}_{u}, \mathfrak{h}\right\}$. That is

(i) $\tau E_{\alpha}=-E_{-\alpha}$,

(ii) $\left[E_{\alpha}, E_{-\alpha}\right]=H_{\alpha}$.

Then $\sigma E_{\alpha}=-E_{-\alpha}$ for $\alpha \in \Delta_{K}, \sigma E_{\alpha}=E_{-\alpha}, \alpha \in \Delta_{P}$.

LemMA 5.4. Let $\alpha_{1}, \ldots, \alpha_{j}, \beta_{1}, \ldots, \beta_{j} \in \Delta_{P}^{+}$then $f(z)=$ $\xi_{z \Lambda_{1}}\left(E_{\beta_{1}} \cdots E_{\beta_{j}} E_{-\alpha_{1}} \cdots E_{-\alpha_{j}}\right)$ is a polynomial in $z$ of degree at most $j$. Furthermore $f$ is of degree $j$ if and only if there is a permutation of $\{1, \ldots, j\}$, $\delta$, so that $\alpha_{\delta i}=\beta_{i}$. Finally, if there is such $a \delta$ the coefficient of $z^{j}$ in $f$ is positive.

Proof. By induction on $j$. If $j=1$ then $\xi_{z \Lambda_{1}}\left(E_{\beta_{1}} E_{-\alpha_{1}}\right)=\xi_{z \Lambda_{1}}\left(\left[E_{\beta_{1}}, E_{-\alpha_{1}}\right]\right)$. If $\beta_{1} \neq \alpha_{1}$ then $\left[E_{\beta_{1}}, E_{-\alpha_{1}}\right] \in\left[\mathfrak{f}_{c}, \mathfrak{f}_{c}\right]$ and $\xi_{z \Lambda_{1}}\left(\left[\mathfrak{f}_{c}, \mathfrak{f}_{c}\right]\right)=0$. If $\beta_{1}=\alpha_{1}$ then $\xi_{z \Lambda_{1}}\left(E_{\beta_{1}}, E_{-\alpha_{1}}\right)=z \Lambda_{1}\left(H_{\beta_{1}}\right)$. Since $\Lambda_{1}\left(H_{\beta}\right)>0$ for $\beta \in \Delta_{P}^{+}$the result follows for $j=1$. 
Suppose the result is true for $1<j<k-1$. Suppose $j=k$. Then

$$
\begin{aligned}
\xi_{z \Lambda_{1}} & \left(E_{\beta_{1}} \cdots E_{\beta_{k}} E_{-\alpha_{1}} \cdots E_{-\alpha_{k}}\right) \\
& =\sum_{i=1}^{k} \xi_{z \Lambda_{1}}\left(E_{\beta_{1}} \cdots E_{\beta_{k-1}} E_{-\alpha_{1}} \cdots\left[E_{\beta_{k}}, E_{-\alpha_{i}}\right] \cdots E_{-\alpha_{k}}\right) .
\end{aligned}
$$

If $\beta_{k} \neq \alpha_{j}$ for any $j$ then $\left[E_{\beta_{k}}, E_{-\alpha}\right] \in\left[\mathfrak{f}_{c}, \mathfrak{f}_{c}\right]$ for all $j$. Since $\left[\mathfrak{f}_{c}, \mathfrak{f}_{c}\right] \xi_{z \Lambda_{1}}=0$ we see that if $\beta_{k} \neq \alpha_{i}$ for any $i$ then the right-hand side of (*) is of the form

$\sum_{i=1}^{k} \sum_{j=i+1}^{k} \xi_{z \Lambda_{1}}\left(E_{\beta_{1}} \cdots E_{\beta_{k-1}} E_{-\alpha_{1}} \cdots E_{-\alpha_{i+1}} \cdots\right.$

$$
\left.\left[\left[E_{\beta_{k}}, E_{-\alpha_{i}}\right], E_{-\alpha_{j}}\right] \cdots E_{-\alpha_{k}}\right)
$$

But $\left[\left[E_{\beta_{k}}, E_{-\alpha_{i}}\right], E_{-\alpha_{j}}\right] \neq 0$ only if $\beta_{k}-\alpha_{i}-\alpha_{j} \in \Delta_{P}^{+}$. Thus if $\beta_{k} \neq \alpha_{i}$ for any $i$ we see that $f$ is of degree at most $k-1$ by the inductive hypothesis.

We note that the argument above also shows that the terms in the sum on the right-hand side of (*) with $\alpha_{i} \neq \beta_{k}$ are polynomials of degree $k-1$ or less in $z$. We may relabel so that $\alpha_{1}, \ldots, \alpha_{s}=\beta_{k}, \alpha_{s+j} \neq \beta_{u}, j=1, \ldots, k-s$. Hence

$$
f(z)=\sum_{i=1}^{s} \xi_{z \Lambda_{1}}\left(E_{\beta_{1}} \cdots E_{\beta_{k-1}} E_{-\alpha_{1}} \cdots\left[E_{\beta_{k}}, E_{-\alpha_{i}}\right] \cdots E_{-\alpha_{k}}\right)+h(z)
$$

with $h(z)$ a polynomial of degree at most $k-1$. But then

$$
\begin{aligned}
f(z)= & \sum_{i=1}^{s} \xi_{z \Lambda_{1}}\left(E_{\beta_{1}} \cdots E_{\beta_{k-1}} E_{-\alpha_{1}} \cdots E_{-\alpha_{i-1}} H_{\beta_{k}} E_{-\alpha_{i+1}} \cdots E_{-\alpha_{k}}\right) \\
= & \sum_{i=1}^{s}\left(z \Lambda_{1}\left(H_{\beta_{k}}\right)-\sum_{j=i+1}^{k}\left\langle\alpha_{j}, \beta_{k}\right\rangle\right) \\
& \cdot \xi_{z \Lambda_{1}}\left(E_{\beta_{1}} \cdots E_{\beta_{k-1}} E_{-\alpha_{2}} \cdots E_{-\alpha_{k}}\right) .
\end{aligned}
$$

Since $\Lambda_{1}\left(H_{\beta_{k}}\right)>0$ the result now follows from the inductive hypothesis. Q.E.D.

LEMMA 5.5. $P_{k_{1}, \ldots, k_{r}}$ is a polynomial of degree $t=\sum i k_{i}$. Furthermore $P_{k_{1}, \ldots, k_{r}}(z)=C_{k_{1} \ldots k_{r}} z^{t}+$ lower order terms and $(-1)^{t} C_{k_{1} \ldots k_{r}}>0$.

Proof. Let $\lambda=\sum k_{i} \lambda_{i}$. Let $w=u_{1}^{k_{1}} \cdot \cdots u_{r}^{k_{r}}$. Then $w=$ $\sum a_{\delta_{1}} \ldots \delta_{t} E_{-\delta_{1}} \cdots E_{-\delta_{t}}$ the sum taken over all $\delta_{1}, \ldots, \delta_{t} \in \Delta_{P}^{+}, \Sigma \delta_{i}=-\lambda$ and distinct up to permutation. $\eta(w)=(-1)^{t} \Sigma \bar{a}_{\delta_{1}} \cdots \delta_{1} E_{\delta_{1}} \cdots E_{\delta_{i}}$. Thus

$$
(-1)^{t} P_{k_{1}, \ldots, k_{r}}(z)=\sum \bar{a}_{\delta_{1}} \cdots \delta_{t} a_{\mu_{1}} \cdots \mu_{r} \xi_{z \Lambda_{1}}\left(E_{\delta_{1}} \cdots E_{\delta_{1}} E_{-\mu_{1}} \cdots E_{-\mu_{t}}\right) \text {. }
$$


Thus modulo polynomials of degree $t-1$ or less

$$
(-1)^{t} P_{k_{1}, \ldots, k_{r}}(z)=\sum\left|a_{\delta_{1}} \cdots \delta_{t}\right|^{2} \xi_{z \Lambda_{1}}\left(E_{\delta_{1}} \cdots E_{\delta_{t}} E_{-\delta_{1}} \cdots E_{-\delta_{t}}\right)
$$

The result now follows from Lemma 5.4.

For the remainder of this section we wish to determine precisely when the polynomials $P_{k_{1}, \ldots, k_{r}}(z)$ are nonnegative. To do this we may assume $\Delta=\Delta^{0}$ (see Lemma 5.3). We make this assumption for the next few results.

LEMMA 5.6. If $1 \leqslant j<k \leqslant l$ then $\left[E_{ \pm \gamma_{k}}, u_{j}\right]=0$.

Proof. $u_{j}=\sum a_{\delta_{1}} \ldots \delta_{j} E_{-\delta_{1}} \cdots E_{-\delta_{j}}$ the sum taken over all distinct $\delta_{1}, \ldots, \delta_{j} \in \Delta_{P}^{+}$so that $\sum \delta_{i}=\sum_{i=1}^{j} \gamma_{i}$. But now if $a_{\delta_{1}} \ldots \delta_{j} \neq 0$ then $\delta_{i}=\frac{1}{2}\left(\gamma_{t_{i}}\right.$ $+\gamma_{s_{i}}$ ), $1<t_{i}<s_{i}<r$. (We are assuming that $\Delta=\Delta^{0}$ !) But $\Sigma \delta_{i}=\Sigma_{i=1}^{j} \gamma_{i}$. Hence $1<t_{i}, s_{i}<j$. If $k>j$ then $\left[E_{ \pm \gamma_{k}}, E_{-\delta_{i}}\right] \neq 0$ only if $\pm \gamma_{k}-\delta_{i} \in \Delta$. But $\left.\left( \pm \gamma_{k}-\delta_{i}\right)\right|_{\mathfrak{h}^{-}}= \pm \gamma_{k}-\frac{1}{2}\left(\gamma_{t_{i}}+\gamma_{s_{i}}\right)$ with $t_{i}, s_{i}<k$. This is not one of the forms of $\$ 2$ (iv). Q.E.D.

LEMMA 5.7. If $k>0$ and $E_{\gamma_{r}} u_{r}^{k} \xi_{z \Lambda_{1}}=0$ then $u_{r}^{k} \xi_{z \Lambda_{1}}=0$.

Proof. [ $\left.\mathfrak{f}_{c}, \mathfrak{f}_{c}\right] u_{r}^{k} \xi_{z \Lambda_{1}}=0$. (We are assuming that $\Delta^{0}=\Delta$. In this case $\left\langle\lambda_{r}, \alpha\right\rangle=0$ if $\alpha \in \Delta_{K}$.) Suppose that $E_{\gamma_{r}} u_{r}^{k} \xi_{z \Lambda_{1}}=0$. Then if $Z \in\left[\mathfrak{f}_{c}, \mathfrak{f}_{c}\right]$ we see $0=Z E_{\gamma_{r}} u_{r}^{k} \xi_{z \Lambda_{1}}=\left[Z, E_{\gamma_{r}}\right] u_{r}^{k} \xi_{z \Lambda_{1}}$. Hence $\left(\operatorname{ad} U\left(\left[k_{c}, k_{c}\right]\right) E_{\gamma_{r}}\right) u_{r}^{k} \xi_{z \Lambda_{1}}=0$. But then $\mathfrak{p}^{+} u_{r}^{k} \xi_{z} \Lambda_{1}=0\left(\gamma_{r}\right.$ is the highest root). Since $\mathfrak{n}_{K}^{+} u_{r}^{k} \xi_{z \Lambda_{1}}=0$ we see $\mathfrak{n}^{+}$. $u_{r}^{k} \xi_{z \Lambda_{1}}=0$. But then $u_{r}^{k} \xi_{z \Lambda_{1}}=c \xi_{z \Lambda_{1}}$. But $k>0$ hence $c=0$. Q.E.D.

Now $u_{r}=\sum a_{\beta_{1}} \cdots \beta_{r} E_{-\beta_{1}} \cdots E_{-\beta_{r}}$. The sum over $\beta_{1}, \ldots, \beta_{r} \in \Delta_{P}^{+}$, $\sum_{i=1}^{r} \beta_{i}=\sum_{i=1}^{r} \gamma_{i}$. We may clearly assume $\beta_{1} \leqslant \cdots \leqslant \beta_{r}$. Since $\beta_{r}<\gamma_{r}$ we see $u_{r}=v E_{-\gamma_{r}}+w$ with

$$
w=\sum_{\beta_{r} \neq \gamma_{r}} a_{\beta_{1} \cdots \beta_{r}} E_{-\beta_{1}} \cdots E_{-\beta_{r}}, \quad v \in U\left(\mathfrak{p}_{r-1}^{-}\right) .
$$

Here $\mathfrak{p}_{r-1}^{-}=\sum \mathfrak{g}_{\alpha}$ where $\left.\alpha\right|_{\mathfrak{h}^{-}}=\frac{1}{2}\left(\gamma_{i}+\gamma_{j}\right), i, j \leqslant r-1$. The argument of the proof of Lemma 5.6 implies that $\left[E_{\gamma_{r}}, u_{r}\right]=v H_{\gamma_{r}}+\left[E_{\gamma_{r}}, w\right]$. Furthermore by the form of $w$ we see easily that

$$
\left[E_{\gamma_{r}}, w\right]=w_{1}+\sum_{\alpha \in \Delta_{k}^{+}} w_{\alpha} E_{\alpha}, \quad w_{1}, w_{\alpha} \in U\left(\mathfrak{p}^{-}\right)
$$

Now if $Z \in \mathfrak{n}_{K}^{+},\left[Z, E_{\gamma_{r}}\right]=0$. Thus if $Z \in \mathfrak{n}_{K}^{+}$

$$
\begin{aligned}
0= & {\left[Z,\left[E_{\gamma_{r}}, u_{r}\right]\right]=[Z, v] H_{\gamma_{r}}+v\left[Z, H_{\gamma_{r}}\right]+\left[Z, w_{1}\right] } \\
& +\sum_{\alpha \in \Delta_{k}^{+}}\left[Z, w_{\alpha}\right] E_{\alpha}+\sum_{\alpha \in \Delta_{K}^{+}} w_{\alpha}\left[Z, E_{\alpha}\right] \\
= & {[Z, v] H_{\gamma_{r}}+\left[Z, w_{1}\right]+\sum_{\alpha \in \Delta_{k}^{+}} v_{\alpha} E_{\alpha}, \quad v_{\alpha} \in U\left(p^{-}\right) . }
\end{aligned}
$$


Hence $[Z, v]=\left[Z, w_{1}\right]=0$. But it is clear that $[h, v]=\left(\lambda_{r}+\gamma_{r}\right)(h) v,\left[h, w_{1}\right]$ $=\left(\lambda_{r}+\gamma_{r}\right)(h) w_{1}$. Since $\lambda_{r}+\gamma_{r}=\lambda_{r-1}$ we see that $v=c_{1} u_{r-1}, w_{1}=c_{2} u_{r-1}$. Hence $u_{r}=c_{1} u_{r-1} E_{-\gamma_{r}}+w$, with $w$ as above.

LemMa 5.8. Let the notation be as above. Then $c_{1} \neq 0$.

Proof. We first observe that if $K_{c}$ is the group of automorphisms of $\mathfrak{g}_{c}$ generated by the $\exp (\operatorname{ad} X)$ for $X \in \mathfrak{f}_{c}$. Then $\operatorname{Ad}\left(K_{c}\right)\left(E_{-\gamma_{1}}+\cdots+E_{-\gamma_{r}}\right)$ is open and dense in $\mathfrak{p}_{c}^{-}$. This can be seen in several ways. The easiest is to note that if $\mathfrak{a}=\sum_{i=1}^{r} \mathbf{R}\left(E_{\gamma_{i}}+E_{-\gamma_{i}}\right)$ then $\mathfrak{a}$ is maximal abelian in $\mathfrak{p}$. Then use the fact that $\operatorname{Ad}(K) \mathfrak{a}=\mathfrak{p}$ (here $K \subset K_{c}$ is the subgroup generated by the $\exp (\operatorname{ad} X), X \in \mathfrak{l})$.

But then $\left(E_{-\gamma_{1}}+\cdots+E_{-\gamma_{r}}\right)^{r}$ is a cyclic vector for $U^{r}\left(\mathfrak{p}^{-}\right)$as a representation of $\xi_{c}$. Hence if $\left(E_{-\gamma_{r}}+\cdots+E_{-\gamma_{r}}\right)^{r} \cdot \xi_{\Lambda_{1}}=0$ then $U^{r}\left(\mathfrak{p}^{-}\right) \xi_{\Lambda_{1}}=$ 0. But $-\Lambda_{1}$ is the lowest weight of $V^{\Lambda_{1}}\left(\operatorname{dim} V^{\Lambda_{1}}<\infty\right)$ and since $\Delta^{0}=\Delta$, $\Lambda_{1}=\frac{1}{2}\left(\gamma_{1}+\cdots+\gamma_{r}\right)$. Thus $U^{0, \ldots, 1}\left(\mathfrak{p}^{-}\right) \xi_{\Lambda_{1}} \neq 0$. But $U_{1}^{k_{1}} \cdots U_{r}^{k_{r}} \cdot \xi_{\Lambda_{1}}=$ 0 if $\Sigma i k_{i}=r, k_{i} \neq 1$. Hence $U^{r}\left(\mathfrak{p}^{-}\right) \xi_{\Lambda_{1}}=V_{0,0, \ldots, 0,1}^{\Lambda_{1}}$. Also $2\left\langle\Lambda_{1}, \gamma_{j}\right\rangle /\left\langle\gamma_{j}, \gamma_{j}\right\rangle$ $=1$ thus $E_{-\gamma_{j}}^{2} \xi_{\Lambda_{1}}=0, j=1, \ldots, r$. Let $Q: U^{r}\left(\mathfrak{p}^{-}\right) \rightarrow U^{0, \ldots, 0,1}\left(\mathfrak{p}^{-}\right)$be the $\mathfrak{f}_{c}$ invariant projection. Then since

$$
\left(E_{-\gamma_{r}}+\cdots+E_{-\gamma_{r}}\right)^{r}=\sum \frac{r !}{m_{1} ! \cdots m_{r} !} E_{-\gamma_{1}}^{m_{1}} \cdots E_{-\gamma_{r}}^{m_{r}}
$$

we see $Q\left(E_{-\gamma_{1}}^{m_{1}} \cdots E_{-\gamma_{r_{1}}}^{m_{1}}\right)=0$ if some $m_{j} \neq 1$. Hence $Q\left(E_{-\gamma_{1}} \cdots E_{-\gamma_{r}}\right)=$ $c u_{r}, c \neq 0$. This clearly implies $c_{1} \neq 0$. Q.E.D.

We may thus assume

$$
u_{r}=u_{r-1} E_{-\gamma_{r}}+w_{r}, \quad w_{r}=\sum_{\substack{\beta_{1}<\cdots<\beta_{r} \\ \Sigma \beta_{i}=\sum \beta_{i} \\ \beta_{r} \neq \gamma_{r}}} a_{\beta_{1}} \cdots \beta_{r} E_{\beta_{1}} \cdots .
$$

LeMMA 5.9.

$$
\left[E_{\gamma_{r}}, u_{r}\right]=u_{r-1} H_{\gamma_{r}}+\frac{1}{4}\left\langle\gamma_{r}, \gamma_{r}\right\rangle c_{r} u_{r-1}+\sum_{\alpha \in \Delta_{K}^{+}} w_{\alpha} E_{\alpha}
$$

with $w_{\alpha} \in U\left(\mathfrak{p}^{-}\right)$and $c_{r}$ is the order of $C_{r}=\left\{\alpha \in \Delta_{K}^{+}|\alpha|_{\mathfrak{h}^{-}}=\frac{1}{2}\left(\gamma_{r}-\gamma_{j}\right)\right.$ for some $j<r\}$.

Proof.

$$
w_{r}=\sum_{\substack{\beta_{1}<\ldots<\beta_{r}<\gamma_{r} \\ \Sigma \beta_{i}=\Sigma \gamma_{i}}} a_{\beta_{1} \cdots \beta_{r}} E_{-\beta_{1}} \cdots E_{-\beta_{r}} .
$$

But then $\left.\beta_{r-1}\right|_{\mathfrak{h}^{-}}=\frac{1}{2}\left(\gamma_{r}+\gamma_{j}\right),\left.\beta_{r}\right|_{\mathfrak{h}^{-}}=\frac{1}{2}\left(\gamma_{r}+\gamma_{i}\right)$ with $j<i<r$. Hence

$$
w_{r}=\sum_{\substack{\alpha, \beta \in C_{r} \\ \alpha<\beta}} Z_{\alpha, \beta}\left[E_{\beta}, E_{-\gamma_{r}}\right]\left[E_{\alpha}, E_{-\alpha}\right]
$$


with $z_{\alpha, \beta} \in U\left(\mathfrak{p}_{r-1}^{-}\right)$(see the end of $\$ 2$ for notation). Now if $\alpha \in C_{r}, \beta \in$ $\Delta_{r-1, p}^{+}$then $\alpha-\left.\beta\right|_{\mathfrak{h}}$ - is not of one of the possible forms for a restriction of a root to $\mathfrak{h}^{-}$(see \$2(iii), (iv)). Hence $\left[E_{\alpha}, U\left(\mathfrak{p}_{r-1}^{-}\right)\right]=0$ for $\alpha \in C_{r}$.

Now if $\alpha \in C_{r}$ then $0=\left[E_{\alpha}, u_{r}\right]=u_{r-1}\left[E_{\alpha}, E_{-\gamma_{r}}\right]+\left[E_{\alpha}, w_{r}\right]$. Thus $\left[E_{\alpha}, w_{r}\right]$ $=-u_{r-1}\left[E_{\alpha}, E_{-\gamma_{r}}\right]$ for $\alpha \in C_{r}$. This implies that if $\delta \in C_{r}$

$$
\begin{aligned}
-u_{r-1}\left[E_{\delta}, E_{-\gamma_{r}}\right]= & \sum_{\substack{\alpha, \beta \in C_{r}, \beta \\
\alpha<\beta}} z_{\alpha, \beta}\left[E_{\delta},\left[E_{\beta}, E_{-\gamma_{r}}\right]\right]\left[E_{\alpha}, E_{-\gamma_{r}}\right] \\
& +\sum_{\substack{\alpha, \beta \in C_{r} \\
\alpha<\beta}} z_{\alpha, \beta}\left[E_{\beta}, E_{-\gamma_{r}}\right]\left[E_{\delta},\left[E_{\alpha}, E_{-\gamma_{r}}\right]\right] .
\end{aligned}
$$

We therefore see that

(i) If $\delta \in C_{r}$ then

$$
\sum_{\substack{\beta>\delta \\ \beta \in C_{r}}} z_{\alpha, \beta}\left[E_{\delta},\left[E_{\beta}, E_{-\gamma_{r}}\right]\right]+\sum_{\substack{\delta>\alpha \\ \alpha \in C_{r}}} z_{\alpha, \delta}\left[E_{\delta},\left[E_{\alpha}, E_{-\gamma_{r}}\right]\right]=-u_{r-1} .
$$

If $\beta \in C_{r}$ then $\beta+\gamma_{r} \notin \Delta$ and $\beta-2 \gamma_{r} \notin \Delta$. Thus we see (cf. Wallach [9, Lemma 3.5.12]).

(ii) $\left[E_{\gamma_{r}},\left[E_{\beta}, E_{-\gamma_{r}}\right]\right]=-\frac{1}{2}\left\langle\gamma_{r}, \gamma_{r}\right\rangle E_{\beta}$.

We now compute $\left[E_{\gamma_{r}}, w_{r}\right]$.

$$
\begin{gathered}
=\sum_{\substack{\alpha<\beta \\
\alpha, \beta \in C_{r}}} z_{\alpha, \beta}\left\{\left[E_{\gamma_{r}},\left[E_{\beta}, E_{-\gamma_{r}}\right]\right]\left[E_{\alpha}, E_{-\gamma_{r}}\right]\right. \\
\left.+\left[E_{\beta}, E_{-\gamma_{r}}\right]\left[E_{\gamma_{r}},\left[E_{\alpha}, E_{-\gamma_{r}}\right]\right]\right\} \\
=-\frac{1}{2} \sum_{\substack{\alpha<\beta \\
\alpha, \beta \in C_{r}}} z_{\alpha, \beta}\left\{E_{\beta}\left[E_{\alpha}, E_{-\gamma_{r}}\right]+\left[E_{\beta}, E_{-\gamma_{r}}\right] E_{2}\right\} \\
=-\frac{1}{2}\left\langle\gamma_{r}, \gamma_{r}\right\rangle \sum_{\substack{\alpha<\beta \\
\alpha, \beta \in C_{r}}} z_{\alpha, \beta}\left[E_{\beta},\left[E_{\alpha}, E_{-\gamma_{r}}\right]\right]+\sum_{\delta \in \Delta_{k}} v_{\delta} E_{\delta}
\end{gathered}
$$

with $v_{\delta} \in U\left(p^{-}\right)$.

If $\alpha, \beta \in C_{r}$ then $\alpha+\beta \notin \Delta$. Hence if $\alpha, \beta \in C_{r},\left[E_{\beta},\left[E_{\alpha}, E_{-\gamma_{r}}\right]\right]=$ $\left[E_{\alpha},\left[E_{\beta}, E_{-\gamma_{r}}\right]\right]$. Hence (i) says

(iii) $\Sigma_{\alpha<\beta, \alpha, \beta \in C_{r}} z_{\alpha, \beta}\left[E_{\beta},\left[E_{\alpha}, E_{-\gamma_{r}}\right]\right]=-\frac{1}{2} u_{r-1}$.

This combined with the above computation says

$$
\left[E_{\gamma_{r}}, w_{r}\right]=\frac{1}{4}\left\langle\gamma_{r}, \gamma_{r}\right\rangle c_{r} u_{r-1}+\sum_{\delta \in \Delta_{K}^{+}} v_{\delta} E_{\delta}
$$

$c_{r}$ the order of $C_{r}$.

Since $\left[E_{\gamma_{r}}, u_{r-1} E_{-\gamma_{r}}\right]=u_{r-1} H_{\gamma_{r}}$ the result now follows. Q.E.D. 
We can now prove our main result. We return to the original assumptions on $\mathfrak{g}$ (that is we do not necessarily assume that $\Delta^{0}=\Delta$ ).

THEOREM 5.10. Let for $1 \leqslant j \leqslant r$, $c_{j}$ be the order of $C_{j}=\left\{\alpha \in \Delta_{K}^{+}|\alpha|_{\mathfrak{h}^{-}}\right.$ $\left.=\frac{1}{2}\left(\gamma_{j}-\gamma_{i}\right), i<j\right\}$. Then $V^{z \Lambda_{1}}$ is infinitesimally unitary if and only if $z<$ $-\frac{1}{2} c_{r}$ or $z=-\frac{1}{2} c_{j}$ for some $1 \leqslant j \leqslant r$. (Note $c_{1}=0$ and $V^{0}$ is the trivial representation.)

Proof. We must find the set of all $z$ so that $P_{k_{1}, \ldots, k_{r}}(z) \geqslant 0$ for all $k_{1}, \ldots, k_{r}>0, k_{i} \in \mathbf{Z}$. We first note that if $t=\Sigma i k_{i}$ then $P_{k_{1}, \ldots, k_{r}}(z)=$ $C_{k_{1}}, \ldots, k_{r} z^{t}+$ lower order terms and $(-1)^{t} C_{k_{1}, \ldots, k_{r}}>0$. This implies that if $z_{k_{1}, \ldots, k_{r}}=\min \left\{z \mid P_{k_{1}, \ldots, k_{r}}(z)=0\right\}$ then if $z<z_{k_{1}, \ldots, k_{r}}, P_{k_{1}, \ldots, k_{r}}(z)>0$.

To compute the $P_{k_{1}}, \ldots, k_{r}(z)$ we may assume $\Delta=\Delta^{0}$. For the remainder of the proof we do this. $P_{k_{1}, \ldots, k_{r}}(z)=0$ if and only if $u_{1}^{k_{1}} \cdots u_{r}^{k_{r}} \cdot \xi_{z_{1}}=0$. 0 .

(1) If $P_{k_{1}, \ldots, k_{r}}(z)=0, k_{r}>0$ and $z<-\frac{1}{2} c_{r}$ then $P_{k_{1}, \ldots, k_{r-1}+1, k_{r}-1}(z)=$

Indeed, $P_{k_{1}, \ldots, k_{r}}(z)=0$ if and only if $u_{1}^{k_{1}} \cdots u_{r}^{k_{r}} \xi_{z \Lambda_{1}}=0$. Now

$$
E_{\gamma_{1}} u_{1}^{k_{1}} \cdots u_{r}^{k_{r}} \xi_{z \Lambda_{1}}=u_{1}^{k_{1}} \cdots u_{r-1}^{k_{r-1} E_{\gamma_{r}}} u_{r}^{k_{r}} \xi_{z \Lambda_{1}}
$$

(by Lemma 5.6)

$$
\begin{aligned}
= & \sum_{i=1}^{k_{r}} u_{1}^{k_{1}} \cdots u_{r-1}^{k_{r-1}}\left[E_{\gamma_{r}}, u_{r}\right] u_{r}^{i-1} \xi_{z_{1}} \\
= & \sum_{i=1}^{k_{r}} u_{1}^{k_{1}} \cdots u_{r-1}^{k_{r-1}} u_{r}^{k_{r-i}}\left(u_{r-1} H_{\gamma_{r}}+\frac{1}{4} c_{r}\left\langle\gamma_{r}, \gamma_{r}\right\rangle u_{r-1}\right) u_{r}^{i-1} \xi_{z_{1}} \\
= & \left(-\frac{k_{r}\left(k_{r}-1\right)}{2}\left\langle\gamma_{r}, \gamma_{r}\right\rangle+z \Lambda_{1}\left(H_{\gamma_{r}}\right) k_{r}+\frac{1}{4} c_{r}\left\langle\gamma_{r}, \gamma_{r}\right\rangle k_{r}\right) \\
& \cdot u_{1}^{k_{1}} \cdots u_{r-1}^{k_{r-1}+1} u_{r}^{k_{r}-1} \cdot \xi_{z_{1} \Lambda} .
\end{aligned}
$$

Now

$$
\Lambda_{1}\left(H_{\gamma_{r}}\right)=\Lambda_{1}\left(H_{\gamma_{1}}\right)=\frac{1}{2}\left\langle\gamma_{1}, \gamma_{1}\right\rangle=\frac{1}{2}\left\langle\gamma_{r}, \gamma_{r}\right\rangle
$$

Hence if $P_{k_{1}, \ldots, k_{r}}(z)=0, k_{r}>0$, then

$$
k_{r} \frac{\left\langle\gamma_{r}, \gamma_{r}\right\rangle}{2}\left\{-\left(k_{r}-1\right)+z+\frac{1}{2} c_{r}\right\} u_{1}^{k_{1}} \cdots u_{r-1}^{k_{r-1}+1} u_{r}^{k_{r}-1} \xi_{z \Lambda_{1}}=0 .
$$

If $z<-\frac{1}{2} c_{r}$ then $u_{1}^{k_{1}} \cdots u_{r-1}^{k_{r-1}+1} u_{r}^{k_{r}-1} \xi_{z \Lambda_{1}}=0$ as asserted.

Arguing by induction we therefore see

(2) If $P_{k_{1}, \ldots, k_{r}}(z)=0$ and $z<-\frac{1}{2} c_{r}$ then $P_{k_{1}, \ldots, k_{r-1}+k_{r}, 0}(z)=0$.

Now Lemmas 2.4 and 2.5 imply that $P_{k_{1}, \ldots, k_{j}, 0, \ldots, 0}(z)$ for $j<r$ are computable in $g_{j}$. It is easily proved that $0=c_{1}<c_{2}<\cdots<c_{r}$. Thus we find by induction 
(3) $P_{k_{1}, \ldots, k_{r}}(z) \neq 0$ if $z<-\frac{1}{2} c_{r}$ and $k_{i} \in \mathbf{Z}, k_{i} \geqslant 0$.

(3) combined with the observations at the beginning of the proof implies

(4) If $z<-\frac{1}{2} c_{r}$ then $P_{k_{1}, \ldots, k_{r}}(z)>0$, for all $k_{i} \in \mathbf{Z}, k_{i}>0$.

We next observe

(5) Let $q_{j}=P_{\delta_{j 1}}, \ldots, \delta_{j r}$, the usual Kronecker delta. Then $q_{j}(z)=a_{j} \Pi_{i=1}^{j}\left(z+\frac{1}{2} c_{i}\right)$.

Indeed, $q_{r}(z)=0$ if and only if $u_{r} \cdot \xi_{z \Lambda_{1}}=0$. Lemma 5.9 then says $q_{r}(z)=0$ if and only if $\left(z+\frac{1}{2} c_{r}\right) u_{r-1} \xi_{z \Lambda_{1}}=0$. That is if and only if $\left(z+\frac{1}{2} c_{r}\right) q_{r-1}(z)$ $=0$. The result follows by an induction on $r$.

Clearly $(-1)^{j} a_{j}>0$. This says that if $-\frac{1}{2} c_{r}<z<-\frac{1}{2} c_{r-1}$ then $q_{r}(z)<$ 0 . Similarly if $-\frac{1}{2} c_{j}<z<-\frac{1}{2} c_{j-1}$ then $q_{j}(z)<0$. Also if $z>0$ then $q_{1}(z)<0$. Hence the only $z>-\frac{1}{2} c_{r}$ for which $V^{z \Lambda_{1}}$ can possibly be unitarizable are $z=-\frac{1}{2} c_{j}$ for some $1<j<r$.

But if $z=-\frac{1}{2} c_{j}$ then $P_{k_{1}, \ldots, k_{r}}(z)=0$ if $k_{i}>0$ and $i>j$. One proves as above that if $k_{i}=0, i>j$ and if $z<-\frac{1}{2} c_{j}$ then $P_{k_{1}, \ldots, k_{r}}(z)>0$. Q.E.D.

Corollary 5.11. A necessary and sufficient condition that $P_{k_{1}, \ldots, k_{r}}(z)>0$ for all $k_{1}, \ldots, k_{r} \geqslant 0, k_{i} \in \mathbf{Z}$, is that $z<-\frac{1}{2} c_{r}$.

6. The general case. In this section we consider more general $\Lambda \in \mathfrak{h}^{*}$. If $V^{\Lambda}$ is infinitesimally unitary it is easily checked (cf. Harish-Chandra [1]) that

(1) $2\langle\Lambda, \alpha\rangle /\langle\alpha, \alpha\rangle=n_{\alpha} \in Z, n_{\alpha}>0$ for $\alpha \in \Delta_{K}^{+}$,

(2) $\langle\Lambda, \alpha\rangle<0$ for $\alpha \in \Delta_{p}^{+}$.

If $\Lambda \in \mathfrak{h}^{*}$ satisfies (1) then $\Lambda=\Lambda_{0}+z \Lambda_{1}$ with $\left\langle\Lambda_{0}, H_{\gamma_{1}}\right\rangle=0$ and $\left\langle\Lambda_{0}, \alpha\right\rangle=$ $\langle\Lambda, \alpha\rangle$ for $\alpha \in \Delta_{k}^{+}$.

To study this case we give a different realization of $V^{\Lambda}$. We note that

$$
U=U\left(\mathfrak{f}_{c}\right) \oplus\left(\mathfrak{p}^{-} U+U_{\mathfrak{p}}^{+}\right)
$$

Let $Q: U \rightarrow U\left(\mathfrak{f}_{c}\right)$ be the corresponding projection. If $X \in \mathfrak{f}_{c}, u \in U$, then $X Q(u)=Q(X u) ; Q(u X)=Q(u) X$. (Indeed, $u=Q(u)+Z_{1} w+w Z_{2}, Z_{1} \in$ $\mathfrak{p}^{-}, Z_{2} \in \mathfrak{p}^{+}, X u=X Q(u)+X Z_{1} w+X w Z_{2} . X Z_{1} w=\left[X, Z_{1}\right] w+Z_{1} X w \in$ $\mathfrak{p}^{-} U$.)

Let $W_{\Lambda}$ be the irreducible representation of $f_{c}$ with highest weight $\Lambda$. We note $\operatorname{dim} W_{\Lambda}<\infty$ if and only if $\Lambda$ satisfies (1) above. If $f: U \rightarrow W$ is linear define $(g \cdot f)(x)=f(x g)$, for $x, g \in U$.

If $w \in W$ let $\varepsilon(w)(g)=Q(g) \cdot w . \varepsilon(w): U \rightarrow W_{\Lambda} \cdot \varepsilon(w)\left(\mathfrak{p}^{-} U\right)=0, \mathfrak{p}^{+} \varepsilon(w)$ $=0$. Let $Z^{\Lambda}=U \cdot \varepsilon\left(W_{\Lambda}\right)$. We note that as a $g_{c}$ module $Z^{\Lambda}$ is equivalent with $V^{\Lambda}$. This is an easy consequence of Theorem 3.1 in Wallach [10].

Suppose now $\langle$,$\rangle is a Hermitian form on Z^{\Lambda}$ such that $\langle X \cdot v, w\rangle=$ $-\langle v, X w\rangle$ for $X \in \mathfrak{g}$. Then $\langle x \varepsilon(w), y \varepsilon(v)\rangle=\langle\eta(y) x \varepsilon(w), \varepsilon(v)\rangle$. Now $\eta\left(p^{+}\right)$ $=\mathfrak{p}^{-}, \eta\left(\mathfrak{p}^{-}\right)=\mathfrak{p}^{+}$. Hence we see $\langle\eta(y) x \varepsilon(w), \varepsilon(v)\rangle=\langle Q(\eta(y) x) \varepsilon(w), \varepsilon(v)\rangle$. 
Now if $u \in U\left(\mathfrak{f}_{c}\right)$ then $u \varepsilon(w)=\varepsilon(u w)$ (indeed, $(u \cdot \varepsilon(w))(g)=\varepsilon(w)(g u)=$ $Q(g u) w=Q(g) u w=\varepsilon(u w)(g))$. Hence

(3) $\langle x \varepsilon(w), y \varepsilon(v)\rangle=\langle\varepsilon((Q(\eta(y) x) w), \varepsilon(v)\rangle$.

Now in particular $\langle X \varepsilon(w), \varepsilon(v)\rangle=-\langle\varepsilon(w), X \varepsilon(v)\rangle$. Hence $(w, v)=$ $\langle\varepsilon(w), \varepsilon(v)\rangle$ defines a $\mathfrak{t}$-invariant Hermitian form on $W_{\Lambda}$. Thus $\Lambda=z \Lambda_{1}+$ $\Lambda_{0}, z \in \mathbf{R}$. Since for $z \in \mathbf{R}$ there is a unique (up to scalar multiple) invariant Hermitian form on $W_{\Lambda}$ we see that if we fix a positive definite Hermitian form on $W_{\Lambda},($,$) then up to scalar multiple \langle$,$\rangle is given by$

(4) $\langle x \varepsilon(v), y \varepsilon(w)\rangle=(Q(\eta(y) x) v, w)$.

Let $\tau_{\Lambda}(u) v=Q(u) v$ for $u \in U, v \in W_{\Lambda}$. Then we see

LEMMA 6.1. A necessary and sufficient condition for $V^{\Lambda}\left(\equiv Z^{\Lambda}\right)$ to be $\mathrm{g}$-infinitesimally unitary is that $\tau_{\Lambda}(\eta(y) y)$ be a positive semidefinite operator for $y \in U$.

We now note that if $z \in U\left(\mathfrak{f}_{c}\right)$ then

$$
\tau_{\Lambda}(\eta(y z) y z)=\tau_{\Lambda}(\eta(z) \eta(y) y z)=\tau_{\Lambda}(\eta(z)) \tau_{\Lambda}(\eta(y) y) \tau_{\Lambda}(z) .
$$

But $\left(\tau_{\Lambda}(\eta(z)) v, w\right)=(\eta(z) v, w)=(v, z w)$ for $v, w \in W_{\Lambda}, z \in U\left(\mathfrak{f}_{c}\right)$. Hence if $z \in U\left(\mathfrak{f}_{c}\right)$ then $\tau_{\Lambda}(\eta(z))=\tau_{\Lambda}(z)^{*}$ (Hermitian adjoint). Hence

(5) $\tau_{\Lambda}(\eta(y z) y z)=\tau_{\Lambda}(z)^{*} \tau_{\Lambda}(\eta(y) y) \tau_{\Lambda}(z)$, for $z \in U\left(\mathfrak{f}_{c}\right), y \in U$.

Now $Z^{\Lambda}=U\left(\mathfrak{p}^{-}\right) U\left(\mathfrak{f}_{c}\right) \in\left(W_{\Lambda}\right)=U\left(\mathfrak{p}^{-}\right) \in\left(W_{\Lambda}\right)$. Hence we see

LEMma 6.2. If $\Lambda=z \Lambda_{1}+\Lambda_{0}, z \in \mathbf{R}$. Then a necessary and sufficient condition that $V^{\Lambda}$ be $g$-infinitesimally unitary is that $\tau_{\Lambda}(\eta(u) u)$ be positive semidefinite for $u \in U\left(\mathfrak{p}^{-}\right)$.

LEMMA 6.3. Fix $\Lambda_{0}$ satisfying

(1) $2\left\langle\Lambda_{0}, \alpha\right\rangle /\langle\alpha, \alpha\rangle$ is a nonnegative integer for $\alpha \in \Delta_{K}^{+}$.

(2) $\left\langle\Lambda_{0}, \gamma_{1}\right\rangle=0$.

If $\beta_{1}, \ldots, \beta_{j}, \alpha_{1}, \ldots, \alpha_{j} \in \Delta_{P}^{+}$then

$$
f(z)=\tau_{z \Lambda_{1}+\Lambda_{0}}\left(E_{\beta_{1}} \cdots E_{\beta_{j}} E_{-\alpha_{1}} \cdots E_{-\alpha_{j}}\right)
$$

is a polynomial map of degree $j$ of $\mathbf{C}$ into $\operatorname{End}\left(W_{\Lambda_{0}}\right)\left(W_{\Lambda_{0}} \equiv W_{\Lambda}\right.$ as a representation of $\left.\left[f_{c}, f_{c}\right]\right)$. Furthermore $f$ is of degree $j$ if and only if there is a permutation of $1, \ldots, j, \delta$, so that $\beta_{\delta i}=\alpha_{i}$ for $i=1, \ldots, j$. Under this condition $f(z)=z^{j} A_{j}+\Sigma_{0<i<j} z^{i} A_{i}$ with $A_{j}$ positive definite.

Proof. By induction on $j$. If $j=1$ then $\tau_{z \Lambda_{1}+\Lambda_{0}}\left(E_{\beta_{1}} E_{-\alpha_{1}}\right)=$ $\tau_{z \Lambda_{1}+\Lambda_{0}}\left(\left[E_{\beta_{1}}, E_{-\alpha_{1}}\right]\right)$. If $\beta_{1} \neq \alpha_{1}$ then $\left[E_{\beta_{1}}, E_{-\alpha_{1}}\right] \in\left[\mathfrak{f}_{c}, \mathfrak{f}_{c}\right]$. But then $\tau_{z \Lambda_{1}+\Lambda_{0}}\left(\left[E_{\beta_{1}}, E_{-\alpha_{1}}\right]\right)=\tau_{\Lambda_{0}}\left(\left[E_{\beta_{1}}, E_{-\alpha_{1}}\right]\right)$ which is independent of $z$. If $\beta_{1}=\alpha_{1}$. Then $\tau_{z \Lambda_{1}+\Lambda_{0}}\left(E_{\beta_{1}} \quad E_{-\alpha_{1}}\right)=\tau_{z \Lambda_{1}+\Lambda_{0}}\left(H_{\beta_{1}}\right)$. Now $H_{\beta_{1}}=c H+\tilde{H}_{\beta_{1}}$ with $B\left(\tilde{H}_{\beta_{1}}, H\right)=0$. Thus

$$
c=\frac{\beta_{1}(H)}{B(H, H)}=\frac{1}{B(H, H)} .
$$


Now $\tau_{z \Lambda_{1}+\Lambda_{0}}=\tau_{z \Lambda_{1}} \otimes \tau_{\Lambda_{0}}$. Hence

$$
\begin{aligned}
\left(\tau_{z \Lambda_{1}+\Lambda_{0}}\right)\left(H_{\beta_{1}}\right)=\tau_{z \Lambda_{1}+\Lambda_{0}}\left(\frac{1}{B(H, H)} H+\tilde{H}_{\beta_{1}}\right) \\
=\frac{1}{B(H, H)} \tau_{z \Lambda_{1}}(H) \otimes I+I \otimes\left(\frac{1}{B(H, H)} \tau_{\Lambda_{0}}(H)+\tau_{\Lambda_{0}}\left(\tilde{H}_{\beta_{1}}\right)\right) .
\end{aligned}
$$

Now $\Lambda_{1}(H)>0$. (Indeed $H_{\gamma_{1}}=B(H, H)^{-1} H+\tilde{H}_{\gamma_{1}} \Lambda_{1}\left(H_{\gamma_{1}}\right)=\left\langle\gamma_{1}, \gamma_{1}\right\rangle / 2$. Thus $\left.\Lambda_{1}(H)=\left\langle\gamma_{1}, \gamma_{1}\right\rangle / 2 B(H, H)\right\rangle 0$.) Hence

$$
\tau_{z \Lambda_{1}+\Lambda_{0}}\left(E_{\beta_{1}} E_{-\beta_{1}}\right)=\frac{z}{B(H, H)} I+C, \quad C \text { independent of } z \text {. }
$$

Suppose that the lemma has been proved for $1 \leqslant j<k-1$. Suppose $j=k$. Then

$$
\begin{aligned}
& \tau_{\Lambda}\left(E_{\beta_{1}} \cdots\right.\left.E_{\beta_{k}} E_{-\alpha_{1}} \cdots E_{-\alpha_{k}}\right) \\
&= \sum_{i=1}^{k} \tau_{\Lambda}\left(E_{\beta_{1}} \cdots E_{\beta_{k-1}} E_{-\alpha_{1}} \cdots\left[E_{\beta_{k}}, E_{-\alpha_{i}}\right] \cdots E_{-\alpha_{k}}\right) \\
&= \sum_{i=1}^{k} \sum_{j=i+1}^{k} \tau_{\Lambda}\left(E_{\beta_{1}} \cdots E_{\beta_{k-1}} E_{-\alpha_{1}} \cdots\right. \\
&\left.\quad\left[\left[E_{\beta_{k}}, E_{-\alpha_{i}}\right], E_{-\alpha_{j}}\right] \cdots E_{-\alpha_{k}}\right) \\
&+\sum_{i=1}^{k} \tau_{\Lambda}\left(E_{\beta_{1}} \cdots E_{\beta_{k-1}} E_{-\alpha_{1}} \cdots\right. \\
&\left.E_{-\alpha_{i-1}} E_{-\alpha_{i}} \cdots E_{-\alpha_{k}}\right) \tau_{\Lambda}\left(\left[E_{\beta_{k}} E_{-\alpha_{i}}\right]\right) .
\end{aligned}
$$

All the terms in the first part of the summation are of degree $k-1$ by the inductive hypothesis. Only the terms with $\beta_{k}=\alpha_{i}$ in the second term can be of degree $k$. The case $j=1$ and the inductive hypothesis complete the proof of the lemma. (The details go the same way as the proof of Lemma 5.4.)

LEMMA 6.4. If $u \in U^{j}\left(\mathfrak{p}^{-}\right), v \in U^{k}\left(\mathfrak{p}^{-}\right)$and $j \neq k$ then $\tau_{\Lambda}(\eta(v) u)=0$.

Proof. Let $H$ be as in $\$ 2$. Then ad $H \cdot v=-k v$, and ad $H \cdot u=-j u$.

$$
\begin{aligned}
\tau_{\Lambda}(\eta([H, v]) u) & =\tau_{\Lambda}(\eta(H v) u)-\tau_{\Lambda}(\eta(v H) u) \\
& =\tau_{\Lambda}(\eta(v) H u)-\tau_{\Lambda}(H \eta(v) u) \\
& =\tau_{\Lambda}(\eta(u) H u)=\tau_{\Lambda}(H) \tau_{\Lambda}(\eta(v) u) \\
& =\tau_{\Lambda}(\eta(v) H u)-\tau_{\Lambda}(\eta(v) u) \tau_{\Lambda}(H) \\
& =\tau_{\Lambda}(\eta(v)[\bar{H}, u]) .
\end{aligned}
$$

The lemma now follows. 
We note that Lemmas 6.3 and 6.4 imply (cf. the proof of Lemma 5.5) that if $u \in U(\mathfrak{p}), u \neq 0$ then $\tau_{z \Lambda_{1}+\Lambda_{0}}(\eta(u) u)$ is positive definite if $z$ is sufficiently negative. Thus $\tau_{z \Lambda_{1}+\Lambda_{0}}(\eta(u) u)$ is positive definite at least until the first zero of its determinant. This observation combined with Theorem 5 and Lemma 2.1 of Harish-Chandra [1] implies

TheOREM 6.5 (HARISH-ChaNdRA [1]). If $\Lambda$ satisfies (1) above and $\langle\Lambda+$ $\rho, \beta><0$ for $\beta \in \Delta_{P}^{+}$then $V^{\Lambda}$ is g-infinitesimally unitary.

This result was originally proved by Harish-Chandra by showing that if $\langle\Lambda+\rho, \beta\rangle<0$ for $\beta \in \Delta_{P}^{+}$then $V^{\Lambda}$ is the representation of $g_{c}$ on the $K$-finite vectors of a holomorphic discrete series representation.

We will return to the general question of when $V^{\Lambda}$ is $g$-infinitesimally unitary in a later paper in this series.

7. Relations with the Weil representation. Let $G$ be the metaplectic group $M p(n, \mathbf{R})$. That is, $G$ is a double covering of $\operatorname{Sp}(n, \mathbf{R})(\operatorname{Sp}(2 n, \mathbf{R})$ to some). Recall that $\operatorname{Sp}(n, \mathbf{R})$ is the group of all $2 n \times 2 n$ real matrices satisfying

$$
g J^{t} g=J
$$

with

$$
J=\left[\begin{array}{rr}
0 & I \\
-I & 0
\end{array}\right]
$$

Let $g$ be the Lie algebra of $M p(n, \mathbf{R})$ (or $S P(n, \mathbf{R}))$. Then as is well known $g$ is simple and satisfies the hypotheses of $\$ 2$ (cf. Helgason [3]).

We fix the Cartan involution on $g, \theta(X)=-{ }^{t} X$. Then $f$ is isomorphic with the Lie algebra of the unitary group. In this case $l=r=n$. Furthermore the roots (in the notation of $\$ 2$ ) are given as follows:

(1) $\Delta_{P}^{+}=\left\{\frac{1}{2}\left(\gamma_{i}+\gamma_{j}\right) \mid 1 \leqslant i \leqslant j \leqslant n\right\}$,

(2) $\Delta_{K}^{+}=\left\{\frac{1}{2}\left(\gamma_{i}-\gamma_{j}\right) \mid 1 \leqslant j<i \leqslant n\right\}$.

$\gamma_{1}$ is the unique simple root in $\Delta_{P}^{+}$and $\gamma_{n}$ is the largest root of $\Delta$. If $c_{j}$ is as in Theorem 5.10 then $c_{j}=j-1, j=1, \ldots, n$. In this case Theorem 5.10 becomes

THEOREM 7.1. $V^{z \Lambda_{1}}$ is infinitesimally unitary if and only if $z \leqslant-(n-1) / 2$ or $z=-j / 2, j=1,2, \ldots, n-2$.

The representations $V^{z \Lambda_{1}}, z=-j / 2, j=1,2, \ldots$, have an interesting interpretation in terms of the Weil representation.

We first recall the definition of the Weil representation. Let (, ) be the standard inner product on $\mathbf{R}^{n}$. Let $\mathbf{R}^{2 n}$ be looked upon as column vectors

$$
\left[\begin{array}{l}
x \\
y
\end{array}\right], \quad x, y \in \mathbf{R}^{n}
$$


Define $B\left(\left[\begin{array}{l}x \\ y\end{array}\right],\left[\begin{array}{l}x^{\prime} \\ y^{\prime}\end{array}\right]\right)=\left(x, y^{\prime}\right)-\left(y, x^{\prime}\right)$. Let $H_{n}$ be the Lie group $\mathbf{R}^{2 n} \times \mathbf{R}$ with multiplication

$$
\left(\left[\begin{array}{l}
x \\
y
\end{array}\right], t\right) \cdot\left(\left[\begin{array}{l}
x^{\prime} \\
y^{\prime}
\end{array}\right], t^{\prime}\right)=\left(\left[\begin{array}{l}
x+x^{\prime} \\
y+y^{\prime}
\end{array}\right], t+t^{\prime}+\frac{1}{2} B\left(\left[\begin{array}{l}
x \\
y
\end{array}\right],\left[\begin{array}{l}
x^{\prime} \\
y^{\prime}
\end{array}\right]\right)\right) .
$$

The "standard representation" of $H_{n}$ is given on $L^{2}\left(\mathbf{R}^{n}\right)$ as follows:

$$
\left(T\left(\left(\left[\begin{array}{l}
x \\
y
\end{array}\right], t\right)\right) f\right)(z)=\exp \left(i\left(t+\left(x, z-\frac{1}{2} y\right)\right)\right) f(z-y) .
$$

It is easy to check that $\left(T, L^{2}\left(\mathbf{R}^{n}\right)\right)$ defines an irreducible unitary representation of $H_{n}$.

Let $\mathcal{H}_{n}=T\left(H_{n}\right) \subset U\left(L^{2}\left(\mathbf{R}^{n}\right)\right)$ (the unitary operators on $\left.L^{2}\left(\mathbf{R}^{n}\right)\right)$. Give $\mathcal{H}_{n}$ the subspace topology. One notes that $T: H_{n} /(0, Z) \rightarrow \mathcal{H}_{n}$ is a homeomorphism of topological groups.

Let $G_{0}$ be the set of all $g \in U\left(L^{2}\left(\mathbf{R}^{n}\right)\right)$ satisfying

$$
g T\left(\left[\begin{array}{l}
x \\
y
\end{array}\right], t\right) g^{-1}=T\left(\left[\begin{array}{l}
x^{\prime} \\
y^{\prime}
\end{array}\right], t\right)
$$

for all $\left[\begin{array}{l}x \\ y\end{array}\right] \in \mathbf{R}^{2 n}, t \in \mathbf{R}$. If we set for $g \in G_{0}, \nu(g)\left[\begin{array}{l}x \\ y\end{array}\right]=\left[\begin{array}{l}x^{\prime} \\ y\end{array}\right]$. Then giving $G_{0}$ the subspace topology in $U\left(L^{2}\left(R^{n}\right)\right)$ we see that

(a) $\nu(g) \in S p(n, \mathbf{R})$

(b) $\nu: G_{0} \rightarrow \operatorname{Sp}(n, \mathbf{R})$ is surjective and defines a continuous homomorphism,

(c) $\operatorname{Ker} \nu=\{\lambda I|| \lambda \mid=1\}$.

Let $G_{1}$ be the commutator subgroup of $G_{0}$. Then $\nu: G_{1} \rightarrow \operatorname{Sp}(n, \mathbf{R})$ is a double covering. This gives a realization of $M p(n, \mathbf{R})$. The Weil representation is given by taking an isomorphism $W$ of $M p(n, \mathbf{R})$ with $G_{1}$.

$\left(W, L^{2}\left(\mathbf{R}^{n}\right)\right)$ then defines a representation of $M p(n, \mathbf{R})$.

We note that the space of $C^{\infty}$ vectors of the representation $\left(T, L^{2}\left(R^{n}\right)\right)$ is $\delta\left(\mathbf{R}^{n}\right)$ the Schwartz space of $\mathbf{R}^{n}$. By definition of $G_{0}, W(g): \delta\left(\mathbf{R}^{n}\right) \rightarrow \delta\left(\mathbf{R}^{n}\right)$ for $g \in M p(n, \mathbf{R})$. It is easily checked that $\delta\left(\mathbf{R}^{n}\right)$ is (at least) contained in the space of $C^{\infty}$ vectors for $\left(W, L^{2}\left(\mathbf{R}^{n}\right)\right)$.

Thus $W$ induces a representation of $M p(n, \mathbf{R})$ on $\delta\left(\mathbf{R}^{n}\right)$. We normalize the homomorphism of $\operatorname{Mp}(n, \mathbf{R})$ into $G_{0}$ as follows: let $\mathfrak{n}$ be the subalgebra of $\mathfrak{g}$ consisting of matrices of the form

$$
Z(X)=\left[\begin{array}{cc}
0 & X \\
0 & 0
\end{array}\right], \quad{ }^{t} X=X, \quad X, \quad n \times n,
$$

and $\overline{\mathfrak{n}}$ the subalgebra consisting of matrices of the form

$$
\bar{Z}(X)=\left[\begin{array}{ll}
0 & 0 \\
X & 0
\end{array}\right], \quad{ }^{t} X=X, \quad X, \quad n \times n .
$$


Define for $X$ a real $n \times n$ symmetric matrix $(\mu(X) f)(z)=$ $\exp (i(X z, z) / 2) f(z)$. If $f \in \mathcal{S}\left(\mathbf{R}^{n}\right)$, define

$$
(\mathscr{F} f)(z)=(2 \pi)^{-n / 2} \int_{\mathbf{R}^{n}} e^{-i(z, x)} f(x) d x .
$$

Then

(1) $\mu(X) T(z, t) \mu(X)^{-1}=T(\exp (Z(X)) z, t)$,

(2) $\mathscr{F} T(z, t) \mathscr{F}^{-1}=T(-J z, t)$.

From (1), (2) we find

(3) $\mathscr{F} \mu(X) \mathscr{F}^{-1} T(z, t) \mathscr{F} \mu(X)^{-1} \mathscr{F}^{-1}=T(\exp (\bar{Z}(-X)) z, t)$.

It is not hard to check that $G_{1}$ is the closure in $G_{0}$ of the subgroup generated by the $\mu(X)$ and $\mathscr{F} \mu(X) \mathscr{F}^{-1}, X$ running through the $n \times n$ symmetric matrices.

We normalize $W$ by

(4) $W(\exp (Z(X)))=\mu(X)$,

(5) $W(\exp (\bar{Z}(X)))=\mathscr{F} \mu(-X) \mathscr{F}^{-1}$.

$\left(W, L^{2}\left(\mathbf{R}^{n}\right)\right)$ (also denoted $\left(W_{n}, L^{2}\left(\mathbf{R}^{n}\right)\right)$ ) is called the Weil representation of $M p(n, \mathbf{R})$.

Let for $k \in \mathbf{Z}, k \geqslant 1, M_{n, k}(\mathbf{R})$ be the space of all $n \times k$ matrices. As a vector space $M_{n, k}(\mathbf{R})$ is just $\mathbf{R}^{n k}$. Let $M_{n, k}(\mathbf{R}) \times M_{n, k}(\mathbf{R})$ be the space of columns

$$
\left[\begin{array}{c}
X \\
Y
\end{array}\right], \quad X, Y \in M_{n, k}(\mathbf{R})
$$

Define

$$
B\left(\left[\begin{array}{c}
X \\
Y
\end{array}\right],\left[\begin{array}{c}
X^{\prime} \\
Y^{\prime}
\end{array}\right]\right)=\operatorname{tr}\left({ }^{t} X Y^{\prime}\right)-\operatorname{tr}\left({ }^{t} Y X^{\prime}\right) .
$$

Let $\psi_{k}: S p(n, \mathbf{R}) \rightarrow S p(n k, \mathbf{R})$ be defined by

$$
\psi_{k}(g)\left[\begin{array}{c}
X \\
Y
\end{array}\right]=g\left[\begin{array}{c}
X \\
Y
\end{array}\right]
$$

Then $\psi_{k}$ has a lift, $\tilde{\psi}_{k}: M p(n, \mathbf{R}) \rightarrow M p(n k, \mathbf{R})$. Let $W_{n k}$ be the Weil representation of $M p(n k, \mathbf{R})$. Let $W_{n}^{k}(g)=W_{n k}\left(\tilde{\psi}_{k}(g)\right)$ for $g \in M p(n, \mathbf{R})$. Then $\left(W_{n}^{k}\right.$; $\left.L^{2}\left(M_{n, k}(\mathbf{R})\right)\right)$ is a unitary representation of $M p(n, \mathbf{R})$.

THEOREM 7.2. Let $L_{0}^{2}\left(M_{n, k}(\mathbf{R})\right)=\left\{f \in L^{2}\left(M_{n, k}(\mathbf{R})\right) \mid f(X g)=f(X)\right.$ for $g \in$ $O(k)\}\left(O(k)\right.$ is the orthogonal group of $\left.\mathbf{R}^{k}\right)$. Let $T_{n}^{k}(g)=\left.W_{n}^{k}(g)\right|_{L_{0}^{2}\left(M_{n, k}(\mathbf{R})\right)}$ : Then with an appropriate choice of $\mathfrak{h}$ and $\Delta^{+},\left(T_{n}^{k}, L_{0}^{2}\left(M_{n, k}(\mathbf{R})\right)\right.$ gives a realization of $V^{-(k / 2) \Lambda_{1}} k=1,2, \ldots$ (The choices will be clear in the course of the proof.)

Proof. We will also use the notation $W_{n}$ for the differential of $W_{n}$ on $\delta\left(\mathbf{R}^{n}\right)$. Then 
(1) $W_{n}(Z(X)) f(z)=i(X z, z) f(z) / 2$ for $f \in \mathcal{S}\left(\mathbf{R}^{n}\right)$ and ${ }^{t} X=X, X$ and $n \times n$ matrix.

(2) $\left(W_{n}(Z(X)) f\right)=(i / 2) \sum x_{i j} \partial^{2} f / \partial z_{i} \partial z_{j}$ for $f \in S\left(\mathbf{R}^{n}\right),{ }^{t} X=X$.

We take for $\mathfrak{h}_{*} \subset \mathfrak{f}$ the subalgebra of matrices

$$
h(D)=\left[\begin{array}{cc}
0 & D \\
-D & 0
\end{array}\right]
$$

$D=\operatorname{diag}\left(\lambda_{1}, \ldots, \lambda_{n}\right), \lambda_{i} \in \mathbf{R}\left(\operatorname{diag}\left(\lambda_{1}, \ldots, \lambda_{n}\right)\right.$ is the diagonal matrix with diagonal entries $\left.\lambda_{1}, \ldots, \lambda_{n}\right)$. Let $h_{j}=i h\left(\operatorname{diag}\left(\delta_{1 j}, \ldots, \delta_{n j}\right)\right), j=1, \ldots, n$. Then $W_{n}\left(h_{j}\right)=\frac{1}{2}\left(\partial^{2} / \partial x_{j}^{2}-x_{j}^{2}\right), j=1, \ldots, n . h_{1}, \ldots, h_{n} \operatorname{span} \mathfrak{h}_{\mathbb{R}}=i \mathfrak{h}_{*}$.

We take the lexicographic order on $\Delta$, the roots of $\mathfrak{g}_{c}$ relative to $\mathfrak{h}$ (the complexification of $\mathfrak{h}_{*}$ ), corresponding to the ordered basis $h_{n}, h_{n-1}, \ldots, h_{1}$.

Let $M_{n, k}(\mathbf{R})$ have coordinates $X_{j l}, j=1, \ldots, n, l=1, \ldots, k$. Then it is easily seen that

(3) $W_{n}^{k}\left(h_{j}\right)=\frac{1}{2} \sum_{l=1}^{k}\left(\partial^{2} / \partial X_{j l}^{2}-X_{j l}^{2}\right)$.

Set

$$
A_{j l}^{+}=\frac{i}{2}\left(\frac{\partial}{\partial X_{j l}}+X_{j l}\right), \quad A_{j l}^{-}=\frac{i}{2}\left(\frac{\partial}{\partial X_{j l}}-X_{j l}\right)
$$

on $\delta\left(M_{n, k}(\mathbf{R})\right)$. Then

(4) $\left[A_{j l}^{+}, A_{r s}^{-}\right]=\delta_{j r} \delta_{l s} I$.

(5) $W_{n}^{k}\left(h_{j}\right)=-\sum_{l=1}^{k} A_{j l}^{+} A_{j l}^{-}+(k / 2) I$.

(6) $\left[A_{j l}^{+}, A_{r s}^{+}\right]=\left[A_{j l}^{-}, A_{r s}^{-}\right]=0$.

Let $\phi_{0}(X)=\exp \left(-\frac{1}{2} \operatorname{tr}\left({ }^{t} X X\right)\right)$ and set $\psi_{0}=\left\|\phi_{0}\right\|^{-1} \phi_{0}(\|\ldots\|$ will always denote $L^{2}$ norm). If $J=\left(j_{r s}\right)$ is an $n \times k$ matrix with $j_{r s}>0, j_{r s} \in Z$, define

(7) $\psi_{J}=\left(\Pi_{r s}\left(j_{r s}\right) !\right)^{-1 / 2} \Pi_{r s}\left(A_{r s}^{-}\right)^{j_{r s}} \cdot \psi_{0}$.

Then the theory of Hermite functions implies that the $\psi_{J}$ form an orthonormal basis of $L^{2}\left(M_{n, k}(\mathbf{R})\right)$ (cf. Wiener [12]).

It is easily checked from

(8) $A_{r s}^{+} \phi_{0}=0$

and (4), (6) that

(9) $A_{i l}^{+} A_{i l}^{-} \psi_{J}=\left(j_{i l}+1\right) \psi_{J}$.

\section{Hence}

(10) $W_{n}^{k}\left(h_{i}\right) \psi_{J}=\left(-k / 2-\sum_{l=1}^{k} j_{i l}\right) \psi_{J}$.

This implies that $W_{n}^{k}$ is $\mathfrak{h}$-finite and the weights of $W_{n}^{k}$ are bounded above relative to $\Delta^{+}$. Since $W_{n}^{k}$ is unitary this implies that $\left(W_{n}^{k}, L^{2}\left(M_{n, k}(\mathbf{R})\right)\right.$ splits into a countable direct sum of irreducible unitary representations with highest weights. Using (10) and the fact that relative to the above order of $\Delta^{+}$, $\gamma_{i}\left(h_{j}\right)=2 \delta_{i j}$. To prove the theorem it is enough to show

(11) If $f \in \Sigma_{j} \mathbf{C} \psi_{J}$ (algebraic sum) satisfies

(i) $f(X g)=f(X)$ for $g \in O(k)$, 
(ii) $W_{n}^{k}\left(\mathfrak{n}^{+}\right) f=0$,

then $f=c \phi_{0}, c \in \mathbf{C}$.

Using (1), (2) it is easy to find $W\left(\mathfrak{n}^{+}\right)$.

$$
W\left(\mathrm{n}^{+}\right)=\sum_{i, j=1}^{n} \mathrm{C}\left(\sum_{l=1}^{k} A_{i l}^{+} A_{j l}^{+}\right)+\sum_{1<s<r<n} \mathrm{C}\left(\sum_{l=1}^{k} A_{r l}^{+} A_{s l}^{-}\right) .
$$

Let $\mathcal{H}=\Sigma \mathbf{C} \psi_{J}$ (algebraic sum). If $f \in \mathcal{K}$, then $\phi_{0}^{-1} f$ is a polynomial function on $M_{n, k}(\mathbf{R})$. Let $\mathscr{P}\left(M_{n, k}(\mathbf{R})\right)$ be the space of all polynomial functions on $M_{n, k}(\mathbf{R})$ then clearly $\mathscr{H}=\mathscr{P}\left(M_{n, k}(\mathbf{R})\right) \phi_{0}$. Using this observation (11) reduces to

(13) If $f \in \mathscr{P}\left(M_{n, k}(\mathbf{R})\right)$ and

(i) $\sum_{j=1}^{k} \partial^{2} f / \partial X_{i j} \partial X_{l j}=0, i, l=1, \ldots, n$,

(ii) $\sum_{j=1}^{k} X_{r j} \partial f / \partial X_{i j}=0,1 \leqslant i<r \leqslant n$,

(iii) $f(X g)=f(X)$ for $g \in O(k)$.

Then $f$ is constant.

But after successive polarizations (Weyl [13, p. 5]), $f$ becomes a trace free tensor invariant for $O(k)$. Now Theorems 5, 7E, F, G and $\mathrm{H}$ of Weyl [13, pp. $157,158]$ imply that $f$ must be constant. Q.E.D.

\section{REFERENCES}

1. Harish-Chandra, Representations of semisimple Lie groups. IV, Amer. J. Math. 77 (1955), 743-777. MR 17, 282.

2. __ Representations of semisimple Lie groups. VI, Amer. J. Math. 78 (1956), 564-628. MR 18, 490.

3. S. Helgason, Differential geometry and symmetric spaces, Academic Press, New York, 1962. MR $26 \$ 2986$.

4. R. Hotta and N. R. Wallach, On Matsushima's formula for the Betti numbers of a locally symmetric space (to appear).

5. C. C. Moore, Compactifications of symmetric spaces. II. The Cartan domains, Amer. J. Math. 86 (1964), 358-378. MR $28 \sharp 5147$.

6. H. Rossi and M. Vergue, Analytic continuation of the holomorphic discrete series of a semi-simple Lie group (to appear).

7. W. Schmid, Die Randwerte holomorpher Funktionen auf hermitesch symmetrischen Räumen, Invent. Math. 9 (1969/70), 61-80. MR 41 \$3806.

8. N. R. Wallach, The analytic continuation of the discrete series. I, Trans. Amer. Math. Soc.

9. __ Harmonic analysis on homogeneous spaces, Dekker, New York, 1973.

10. Induced representations of Lie algebras. II, Proc. Amer. Math. Soc. 21 (1969), 161-166. MR $38 \sharp 5871$.

11. __ On maximal subsystems of root systems, Canad. J. Math. 20 (1968), 555-574. MR $38 \# 1135$.

12. N. Wiener, The Fourier integral and certain of its applications, Cambridge Univ. Press, Cambridge, 1933; reprint, Dover, New York, 1959. MR $20 \sharp 6634$.

13. H. Weyl, The classical groups, Princeton Univ. Press, Princeton, N. J., 1946. (1939 ed., MR 1, 42.)

Department of Mathematics, Rutgers University, New Brunswick, New Jersey 08903 